\title{
Krankenhauspolitische Chronik
}

\author{
Elektronisches Zusatzmaterial Die elektronische Version dieses Kapitels enthält Zusatzmaterial, das den \\ Nutzern zur Verfügung steht unter https://doi.org/10.1007/978-3-662-58225-1_15. \\ Dirk Bürger und Christian Wehner \\ (c) Der/die Autor(en) 2019 \\ J. Klauber et al. (Hrsg.), Krankenhaus-Report 2019 \\ https://doi.org/10.1007/978-3-662-58225-1_15
}

\section{Zusammenfassung}

Der Deutsche Bundestag, dessen Abgeordnete im Ausschuss für Gesundheit, das Bundesgesundheitsministerium, die Landesgesundheitsminister und der Bundesrat setzen jährlich neben den gesundheits- auch die krankenhauspolitischen Rahmenbedingungen. Die Gesundheitsexperten der Parteien, diverse Verbände, die (Sozial-)Gerichtsbarkeit und Bundesbehörden sowie politiknahe und wissenschaftliche Institute prägen dabei die öffentliche Diskussion um diese Regelungen. Die Selbstverwaltungspartner auf Bundesebene nutzen die ihnen übertragenen vertraglichen Freiräume, um die medizinische und pflegerische Versorgung in den Krankenhäusern weiterzuentwickeln. Mit der „Krankenhauspolitischen Chronik“liegt eine Übersicht über alle wesentlichen Entscheidungen der Akteure der deutschen Gesundheits- und Krankenhauspolitik vor und informiert über die Aktivitäten in den vergangenen 12 Monaten.

The Deutscher Bundestag, its members of the Health Committee, the Federal Ministry of Health, the state health ministers and the Bundesrat set the health and hospital policy framework conditions every year. The parties' health experts, various associations, the (social) judiciary and federal authorities as well as policyrelated and scientific institutes shape the public discussion about these regulations. The self-governing partners at the federal level use the contractual freedom conferred on them to further develop medical and nursing care in hospitals. The "Hospital Policy Chronicle" provides an overview of all major decisions taken by players in German health and hospital policy and provides information on activities over the past 12 months.

Die Wahl zum 19. Deutschen Bundestag am 24. September 2017 hat nicht nur ein größeres Parlament, sondern auch neue Herausforderungen für alle Verantwortlichen geschaffen. Zunächst freuen konnten sich die Mitglieder der „Freien Demokratischen Partei“ (FDP), die mit 10,7 Prozent die Zeit ihrer außerparlamentarischen Opposition mehr als glückselig beenden konnten. Aber diese Freude verflog recht schnell, da die Option auf ein neues Regierungsbündnis mit CDU/CSU und Grünen platzte. Dass es im Deutschen Bundestag jemals eine Kraft geben würde, die den Raum rechts von der CDU/CSU besetzen könnte, war für viele nicht vorstellbar. Dass es dann sogar der „Alternative für Deutschland“ (AfD) mit 12,6 Prozent der Wählerstimmen gelang, drittstärkste Kraft zu werden, sorgte für erheblichen Frust. Des Wählers Frust zu spüren bekamen sowohl CDU/CSU als auch SPD. Denn beide Volksparteien erzielten bei dieser Bundestagswahl die schlechtesten Ergebnisse und müssen zudem die ungewollte Große Koalition fortsetzen. Eine Fortsetzung ihrer Arbeit gelang zwei führenden Gesundheitspolitikern nicht mehr. Zum einen wurde die bisherige parlamentarische Staatssekretärin Annette Widmann-Mauz (CDU), die sogar kurzfristig als neue Gesundheitsministerin in 
den Medien gehandelt worden war, als Staatsministerin ins Bundeskanzleramt berufen, wo sie nun für Migration, Flüchtlinge und Integration zuständig ist. Zum anderen wurde Gesundheitsminister Hermann Gröhe (CDU) abgelöst. Er hat zwar noch den schwarz-roten Koalitionsvertrag mitentwickelt, aber umsetzen muss ihn nun Jens Spahn (CDU): ein klassischer Rollentausch. Denn zuvor musste Gröhe die Spahn'schen Ideen aus dem Koalitionsvertrag 2013 bis 2017 umsetzen, nun liegt es an Spahn, die Verhandlungsvorgaben von Gröhe mit Leben zu füllen. Hermann Gröhe wird stattdessen das Verhandlungsgeschick seines Nachfolgers aus der Perspektive des Stellvertretenden Fraktionsvorsitzenden der CDU/CSU für Arbeit und Soziales erleben.

Obwohl bereits die vergangene 18 . Wahlperiode des Deutschen Bundestages mit 25 gesundheitspolitischen Gesetzen und diesbezüglichen 18 Rechtsverordnungen sehr arbeitsintensiv war, sind für diese Amtszeit nicht weniger gesundheitspolitische Initiativen zu erwarten. Denn der Koalitionsvertrag „Ein neuer Aufbruch für Europa - Eine neue Dynamik für Deutschland - Ein neuer Zusammenhalt für unser Land" schafft viel Raum für entsprechende dynamische gesetzgeberische Aktivitäten der politischen Verantwortungsträger. Als Schlagworte seien hier nur GKV-Finanzierung, E-Health und Gesundheitswirtschaft oder Sektorübergreifende Versorgung angeführt. Natürlich ist auch dem Krankenhausbereich wieder einmal ein eigener Abschnitt im Koalitionsvertrag gewidmet worden. Allen ist sicherlich auch der Pflege-Azubi Alexander Jorde in Erinnerung geblieben, der die Bundeskanzlerin in der "ARD-Wahlarena“ durch seine Nachfragen zu den Auswirkungen des Fachkräftemangels in der Kranken- und Altenpflege in arge Bedrängnis gebracht hatte. Daher wurde auch ein umfangreiches Maßnahmenpaket „Pflege“ im Koalitionsvertrag vereinbart.

Der Koalitionsvertrag verschafft Bundesgesundheitsminister Jens Spahn nicht nur einen umfangreichen Gestaltungsauftrag, er verfügt zudem auch über einen sehr großen Gestaltungswillen. Dass sich dieser nicht darauf beschränken lässt, einfach nur einen Koalitionsvertrag abzuarbeiten, wurde nicht nur bei Spahns erstem gesundheitspolitischem Gesetzentwurf, dem GKV-Versichertenentlastungsgesetz, deutlich. Mit der „Zwangsweisen
Beitragsabsenkung " bei den gesetzlichen Krankenkassen hat er ein Statement gesetzt. Darüber hinaus bewies Minister Spahn Tatkraft bei seiner zweiten gesundheitspolitischen Initiative, den „Eckpunkten zum Sofortprogramm Kranken- und Altenpflege“. Denn statt wie im Koalitionsvertrag fixiert 8.000 zusätzliche Fachkräftestellen in Pflegeheimen durch die gesetzliche Krankenversicherung (GKV) finanzieren zu lassen, sollen es nun 13.000 Fachkräftestellen sein.

Mit den Eckpunkten wird aber auch die Pflege im Krankenhaus zu einer riesigen „Reform-Baustelle“. Denn die Einführung einer Sonderfinanzierung für das Pflegepersonal im Krankenhaus losgelöst vom DRG-System erfordert eine komplexe Bereinigung der DRG-Fallpauschalen um die Pflegepersonalkosten. Die Finanzierung der Pflegepersonalkosten der Krankenhäuser wird auf eine neue, von den Fallpauschalen unabhängige, krankenhausindividuelle Vergütung der Pflegepersonalkosten ab dem Jahr 2020 umgestellt. Dieses Vorhaben birgt erhebliche Risiken und Nebenwirken sowohl für die Existenz des Gesamtvergütungssystems der DRGs als auch für das einzelne Krankenhaus. Das Pflegekostenvolumen von nicht weniger als 15 Milliarden Euro soll zukünftig nicht mehr über Pauschalen an die Krankenhäuser ausgezahlt und zur „freien Verfügung“ gestellt werden, sondern „(...) auf Grundlage der von den Krankenhäusern geplanten und nachgewiesenen Pflegepersonalausstattung und der entsprechenden Kosten (krankenhausindividuelle Kostenerstattung). Die zweckentsprechende Mittelverwendung ist nachzuweisen. Nicht zweckentsprechend verwendete Mittel sind zurückzuzahlen."

Die Rückkehr zu einer Erstattung der krankenhausindividuellen Kosten für die Pflege 15 Jahre nach Einführung eines leistungsorientierten DRGVergütungssystems stellt nicht weniger als einen Paradigmenwechsel dar. Die Zweckentfremdung von Mitteln zur Finanzierung der Pflege z. B. für Investitionen wäre so aber künftig nicht mehr möglich. Aber auch die Vorgabe der Zweckbindung der finanzierten Mittel für das Pflegepersonal wird für viele Krankenhäuser eher einschränkend wirken. Denn laut Auskunft des Bundesgesundheitsministeriums (BMG) auf eine Kleine Anfrage der Grünen-Bundestagsfraktion überschreitet das DRGErlösvolumen für den Pflegedienst sogar die von 
den Krankenhäusern angegebenen Pflegepersonalkosten um rund 160 Millionen Euro (BundestagsDrs. 19/2321 vom 24.05.2018).

Dass die Bundesländer mit Blick auf die Diskussionen in der Vergangenheit bereit sind, ihre Investitionskostenfinanzierung entsprechend anzupassen, ist stark zu bezweifeln. Die Antwort des BMG auf eine Kleine Anfrage der Grünen-Bundestagsfraktion aus dem Mai 2018 (Bundestags-Drs. 19/2271 vom 23.05.2018) macht deutlich, dass der Fördermittelanteil der Bundesländer seit 1991 von rund 10 Prozent auf ca. 3,5 Prozent des Gesamtumsatzes im Jahr 2015 zurückgegangen ist: mit fatalen Auswirkungen auf die Investitionsfähigkeit der Krankenhäuser. Der bis 2020 verlängerte Krankenhausstrukturfonds wird zwar zusätzliche vier Milliarden Euro an Investitionsmitteln bereitstellen. Diese Aufstockung und Verlängerung wird aber sicherlich nicht ausreichen, um die damit verbundenen politischen Ziele wie z. B. „(...) die Anpassung bestehender Versorgungskapazitäten an den tatsächlichen Versorgungsbedarf sowie die Verbesserung der Wirtschaftlichkeit und Qualität der Krankenhausversorgung" nachhaltig zu gewährleisten. Stattdessen wurde der Strukturfonds nun sogar um neue Fördertatbestände erweitert wie „(...) die telemedizinische Vernetzung von Krankenhäusern“ und darüber hinaus auch die „(...) Investitionen in Ausbildungsstätten", die ebenfalls einen Anteil aus dem Fördertopf für sich beanspruchen werden.

Es wird, falls diese Koalition wirklich über die gesamte Restlaufzeit von noch drei Jahren halten sollte, eine spannende gesundheitspolitische Zeit. Denn eins ist sicher: Auch zukünftig wird es allen Beteiligten nicht an Arbeit mangeln.

Krankenhauspolitische Chronik

\begin{tabular}{|c|c|c|c|}
\hline Termin & Leitbegriff & Vorgang & Legende \\
\hline 30. Juni 2018 & $\begin{array}{l}\text { Selbstver- } \\
\text { waltung }\end{array}$ & $\begin{array}{l}\text { Keine Einigung zu } \\
\text { Pflegepersonalunter- } \\
\text { grenzen }\end{array}$ & $\begin{array}{l}\text { GKV-Spitzenverband (GKV-SV) und Deutsche Kranken- } \\
\text { hausgesellschaft (DKG) können sich nicht auf Pflege- } \\
\text { personaluntergrenzen in sogenannten pflegesensitiven } \\
\text { Bereichen einigen. Diese Vereinbarung hätte bis zum } \\
\text { heutigen Tage erfolgen müssen, damit sie Anfang } 2019 \\
\text { in Kraft treten kann. Offen ist, ob das Bundesministerium } \\
\text { für Gesundheit (BMG) nun eine Ersatzvornahme anstrebt } \\
\text { oder eine „Zwangsschlichtung" einleitet. }\end{array}$ \\
\hline 27. Juni 2018 & Politik & $\begin{array}{l}\text { Regierungsmehrheit } \\
\text { steht - Ausbildungs- } \\
\text { und Prüfungsverord- } \\
\text { nung für die Pflege- } \\
\text { berufe beschlossen }\end{array}$ & $\begin{array}{l}\text { Mit den Stimmen von Union und SPD hat der Ausschuss } \\
\text { für Gesundheit (AfG) des Deutschen Bundestags die } \\
\text { Ausbildungs- und Prüfungsverordnung für die Pflege- } \\
\text { berufe (Bundestags-Drs. 19/2707) mit leichten Verände- } \\
\text { rungen beschlossen. Die Fraktionen von AfD, Die Linke } \\
\text { und Bündnis 90/Die Grünen votierten dagegen, die } \\
\text { FDP-Fraktion enthielt sich. Der Deutsche Bundestag } \\
\text { stimmt am 28. Juni zu. }\end{array}$ \\
\hline 25. Juni 2018 & Politik & $\begin{array}{l}\text { Sorge über Verlust von } \\
\text { Pflegefachwissen }\end{array}$ & $\begin{array}{l}\text { In der öffentlichen Expertenanhörung des AfG zur Aus- } \\
\text { bildungs- und Prüfungsverordnung (Bundestags-Drs. } \\
\text { 19/2707) warnen Sachverständige vor einem Verlust an } \\
\text { Fachwissen in der Kinderkranken- und Altenpflege. So } \\
\text { befürchtet z. B. der Deutsche Pflegerat, dass Altenpflege- } \\
\text { rinnen und Altenpfleger keine sogenannten Vorbehalts- } \\
\text { tätigkeiten ausüben und zukünftig gemeinsam mit einer } \\
\text { Pflegefachkraft arbeiten müssen. Auch die Deutsche } \\
\text { Gesellschaft für Kinder- und Jugendmedizin (DGKJ) } \\
\text { moniert, dass eine selbstständige Pflege von Kindern mit } \\
\text { einer generalistischen Ausbildung ohne Nachqualifika- } \\
\text { tion nicht möglich sei. }\end{array}$ \\
\hline
\end{tabular}


Krankenhauspolitische Chronik

\begin{tabular}{|c|c|c|c|}
\hline Termin & Leitbegriff & Vorgang & Legende \\
\hline 25. Juni 2018 & Politik & $\begin{array}{l}\text { Niedersachsen will } \\
\text { VW-Bußgelder in } \\
\text { Kliniken investieren }\end{array}$ & $\begin{array}{l}\text { Das Land Niedersachsen, so Ministerpräsident Stephan } \\
\text { Weil (SPD), will das Milliarden-Bußgeld von Volkswagen } \\
\text { (VW) vor allem für den Ausbau des schnellen Internets } \\
\text { und für die Unikliniken in Hannover und Göttingen } \\
\text { nutzen. }\end{array}$ \\
\hline 20./21. Juni 2018 & Politik & $\begin{array}{l}\text { Landesgesundheitsmi- } \\
\text { nister fordern bessere } \\
\text { Patienteninformation } \\
\text { bei Krankenhausent- } \\
\text { lassung }\end{array}$ & $\begin{array}{l}\text { Im Rahmen der } 91 . \text { Gesundheitsministerkonferenz (GMK) } \\
\text { in Düsseldorf wurde u. a. auch beraten, wie die Patien- } \\
\text { tenorientierung in der gesundheitlichen Versorgung } \\
\text { verbessert werden kann. In diesem Zusammenhang } \\
\text { befürwortet die GMK, dass Patienten nach jedem Kran- } \\
\text { kenhausaufenthalt einen Patientenbrief erhalten. Denn } \\
\text { bislang gibt es bei der Entlassung nur einen Arztbrief an } \\
\text { den einweisenden Arzt. }\end{array}$ \\
\hline 19. Juni 2018 & $\begin{array}{l}\text { Wissen- } \\
\text { schaft }\end{array}$ & $\begin{array}{l}\text { Immer mehr jüngere } \\
\text { Patienten erhalten ein } \\
\text { künstliches Kniege- } \\
\text { lenk }\end{array}$ & $\begin{array}{l}\text { Laut einer Studie der Bertelsmann-Stiftung erhalten } \\
\text { immer mehr und vor allem auch immer mehr jüngere } \\
\text { Menschen ein künstliches Kniegelenk. Zwischen } 2013 \\
\text { und } 2016 \text { nahm die Zahl solcher Operationen um gut } 18 \\
\text { Prozent auf rund } 169.000 \text { Fälle zu. Bei den unter } 60 \text {-Jäh- } \\
\text { rigen stiegen die Operationszahlen innerhalb dieses } \\
\text { Zeitraumes sogar um } 23 \text { Prozent auf } 33.000 \text { Operationen } \\
\text { an. }\end{array}$ \\
\hline 19. Juni 2018 & $\begin{array}{l}\text { Rechtspre- } \\
\text { chung }\end{array}$ & $\begin{array}{l}\text { Vergütungsanspruch } \\
\text { besteht auch bei } \\
\text { fehlender ver- } \\
\text { tragsärztlicher Einwei- } \\
\text { sung }\end{array}$ & $\begin{array}{l}\text { Das BSG hat entschieden - Az. B } 1 \text { KR 26/17 R -, dass } \\
\text { einem Krankenhaus auch ohne vertragsärztliche Einwei- } \\
\text { sung eines Versicherten eine Vergütung für die Behand- } \\
\text { lung zusteht. Der Vergütungsanspruch für Krankenhaus- } \\
\text { behandlung entstehe, so das BSG in seiner Begründung, } \\
\text { unmittelbar mit der Inanspruchnahme der Leistung, } \\
\text { wenn die Versorgung in einem zugelassenen Kranken- } \\
\text { haus erfolge und erforderlich sowie wirtschaftlich sei. }\end{array}$ \\
\hline 19. Juni 2018 & Politik & $\begin{array}{l}\text { Gutachten zur Über- } \\
\text { und Fehlversorgung in } \\
\text { deutschen Kranken- } \\
\text { häusern }\end{array}$ & $\begin{array}{l}\text { Der Wissenschaftliche Beirat beim Bundesminister der } \\
\text { Finanzen veröffentlicht das Gutachten „Über- und Fehl- } \\
\text { versorgung in deutschen Krankenhäusern“ und schlägt } \\
\text { Änderungen der Krankenhausfinanzierung vor. So sollen } \\
\text { z. B. die Krankenkassen mehr Möglichkeiten bekommen, } \\
\text { mit einzelnen Kliniken Versorgungsverträge zu schlie- } \\
\text { ßen, um so Mindeststandards für die Versorgung und/ } \\
\text { oder niedrigere Preise durchzusetzen. Laut Gutachter } \\
\text { gibt es zu viele kleine und schlecht ausgerüstete Kran- } \\
\text { kenhäuser, die sich zudem nicht kosteneffizient be- } \\
\text { treiben lassen. }\end{array}$ \\
\hline 13. Juni 2018 & $\begin{array}{l}\text { Wissen- } \\
\text { schaft }\end{array}$ & $\begin{array}{l}\text { Anteil an privaten } \\
\text { „Versorgungs" Kran- } \\
\text { kenhäusern steigt } \\
\text { selbst im ländlichen } \\
\text { Raum }\end{array}$ & $\begin{array}{l}\text { Der Anteil von „Versorgungs-“Krankenhäusern in privater } \\
\text { Trägerschaft ist zwischen } 2006 \text { und } 2015 \text { um 6,7 Prozent- } \\
\text { punkte auf } 405 \text { angestiegen, so die die vom RWI heraus- } \\
\text { gegebene Studie „Krankenhäuser in privater Träger- } \\
\text { schaft“. Auch die Anzahl ländlicher Krankenhäuser ist bei } \\
\text { privaten Trägern gestiegen, während sie bei anderen } \\
\text { Trägerschaften rückläufig ist. Im Jahr } 2015 \text { befanden sich } \\
\text { demnach } 38 \text { Prozent der privaten Krankenhäuser im } \\
\text { ländlichen Raum. }\end{array}$ \\
\hline
\end{tabular}


Krankenhauspolitische Chronik

\begin{tabular}{|c|c|c|c|}
\hline Termin & Leitbegriff & Vorgang & Legende \\
\hline 13. Juni 2018 & Politik & $\begin{array}{l}\text { Bundeskabinett be- } \\
\text { schließt Pflegeberufe- } \\
\text { Ausbildungs- und } \\
\text { Prüfungsverordnung }\end{array}$ & $\begin{array}{l}\text { Nach vielfältigen politischen Abstimmungsrunden } \\
\text { beschließt das Bundeskabinett die Pflegeberufe-Ausbil- } \\
\text { dungs- und Prüfungsverordnung (PflAPrV) und hat diese } \\
\text { dem Deutschen Bundestag vor der Zuleitung an den } \\
\text { Bundesrat zur Beschlussfassung vorgelegt. Der Verord- } \\
\text { nungsentwurf regelt insbesondere das Weitere zu den } \\
\text { Mindestanforderungen an die berufliche Pflegeausbil- } \\
\text { dung einschließlich der Zwischenprüfung, die zu vermit- } \\
\text { telnden Kompetenzen und das Verfahren der staatlichen } \\
\text { Prüfungen einschließlich erstmals bundesweit einheit- } \\
\text { licher Rahmenvorgaben für die akademische Pflegeaus- } \\
\text { bildung. }\end{array}$ \\
\hline 7. Juni 2018 & Politik & $\begin{array}{l}\text { Pflegebevollmächtig- } \\
\text { ter setzt auf finanzielle } \\
\text { Anreize für mehr } \\
\text { Pflegepersonal }\end{array}$ & $\begin{array}{l}\text { Der Pflegebevollmächtigte der Bundesregierung, } \\
\text { Andreas Westerfellhaus, stellt sein 5-Punkte-Programm } \\
\text { „Mehr PflegeKRAFT“ für Pflegefachkräfte und Einrich- } \\
\text { tungsbetreiber vor. Er will u. a. durch steuerfreie Prämien } \\
\text { von bis zu 5.000 Euro Anreize setzen, damit dringend } \\
\text { gesuchte Fachkräfte reaktiviert werden können. }\end{array}$ \\
\hline 7. Juni 2018 & $\begin{array}{l}\text { Wissen- } \\
\text { schaft }\end{array}$ & $\begin{array}{l}\text { Krankenhaus-Rating- } \\
\text { Report 2018: } \\
\text { „Personal - Kranken- } \\
\text { häuser zwischen } \\
\text { Wunsch und Wirk- } \\
\text { lichkeit" }\end{array}$ & $\begin{array}{l}\text { Die wirtschaftliche Lage deutscher Krankenhäuser war } \\
\text { im Jahr } 2016 \text { - so die aktuellen Ergebnisse des Kranken- } \\
\text { haus-Rating-Reports } 2018 \text { - wesentlich besser als in den } \\
\text { Jahren zuvor. Nur noch sieben Prozent der Krankenhäu- } \\
\text { ser liegen im „roten Bereich“ mit erhöhter Insolvenzge- } \\
\text { fahr. Ein Problem bleibt aber bestehen: die unzurei- } \\
\text { chenden Fördermittel der Bundesländer für Investiti- } \\
\text { onen. Bei einem jährlichen Investitionsbedarf (ohne } \\
\text { Universitätskliniken) von rund 5,8 Milliarden Euro stellen } \\
\text { die Länder nur Fördergelder in Höhe von 2,8 Milliarden } \\
\text { Euro zur Verfügung. Die jährliche Förderlücke beträgt } \\
\text { somit } 3 \text { Milliarden Euro. }\end{array}$ \\
\hline 5. Juni 2018 & $\begin{array}{l}\text { Wissen- } \\
\text { schaft }\end{array}$ & $\begin{array}{l}\text { Destatis: Mehr } \\
\text { Männer in der Pflege- } \\
\text { ausbildung }\end{array}$ & $\begin{array}{l}\text { Gegenüber } 2006 \text { ist die Zahl der Ausbildungsanfänge- } \\
\text { rinnen und anfänger im Pflegebereich, laut Destatis, um } \\
43 \text { Prozent auf rund } 63.200 \text { angestiegen. Insbesondere } \\
\text { immer mehr Männer entscheiden sich für eine Pflege- } \\
\text { Ausbildung; ihr Anteil an den Auszubildenden nahm um } \\
54 \text { Prozent zu. }\end{array}$ \\
\hline 1. Juni 2018 & Politik & $\begin{array}{l}\text { Breites Bündnis } \\
\text { schlägt wegen Perso- } \\
\text { naluntergrenzen } \\
\text { Alarm }\end{array}$ & $\begin{array}{l}\text { Der Deutsche Pflegerat, ver.di, das Aktionsbündnis } \\
\text { Patientensicherheit sowie viele weitere Verbände und } \\
\text { Interessenvertretungen warnen in einer gemeinsamen } \\
\text { Erklärung vor weiteren Verschlechterungen in der Pflege. } \\
\text { Sie fürchten vor allem, dass sich DKG und GKV-SV auf zu } \\
\text { geringe Vorgaben verständigen, die nicht zu mehr Pati- } \\
\text { entensicherheit in den Krankenhäusern führen. }\end{array}$ \\
\hline 31. Mai 2018 & $\begin{array}{l}\text { Rechtspre- } \\
\text { chung }\end{array}$ & $\begin{array}{l}\text { EuGH: Chefarzt-Kündi- } \\
\text { gung durch katho- } \\
\text { lische Klinik nicht } \\
\text { rechtens }\end{array}$ & $\begin{array}{l}\text { Die Kündigung eines Chefarztes wegen Wiederheirat } \\
\text { durch ein katholisches Krankenhaus war nach Auffas- } \\
\text { sung des EuGH-Generalanwalts nicht rechtens. Für } \\
\text { Kollegen und Patienten sei die Erbringung der medizi- } \\
\text { nischen Leistungen wesentlich und nicht die Frage, ob } \\
\text { der Mediziner geschieden sei und erneut geheiratet } \\
\text { habe. Somit stehe das EU-Verbot der Diskriminierung } \\
\text { wegen Religion einer Kündigung entgegen } \\
\text { (Az.: C-68/17). }\end{array}$ \\
\hline
\end{tabular}


Krankenhauspolitische Chronik

\begin{tabular}{|c|c|c|c|}
\hline Termin & Leitbegriff & Vorgang & Legende \\
\hline 24. Mai 2018 & Politik & $\begin{array}{l}\text { BMG sieht Nachweis- } \\
\text { pflicht für Pflege- } \\
\text { stellen in den Kran- } \\
\text { kenhäusern kritisch }\end{array}$ & $\begin{array}{l}\text { Eine mögliche Nachweispflicht der Krankenhäuser für } \\
\text { die zur Pflege eingesetzten Mittel wird skeptisch ge- } \\
\text { sehen. In einem pauschalierenden Entgeltsystem liege } \\
\text { es grundsätzlich in der betriebswirtschaftlichen Ver- } \\
\text { antwortung der Krankenhäuser, die Betriebsmittel für } \\
\text { eine effektive Versorgung der Patienten einzusetzen, } \\
\text { heißt es in der Antwort (Bundestags-Drs. 19/2321) } \\
\text { auf eine Kleine Anfrage der Fraktion Bündnis 90/Die } \\
\text { Grünen. }\end{array}$ \\
\hline 23. Mai 2018 & Politik & $\begin{array}{l}\text { Mehr Stellen in der } \\
\text { Pflege - vollständige } \\
\text { Refinanzierung der } \\
\text { Pflege-Personalkosten }\end{array}$ & $\begin{array}{l}\text { Bundesgesundheitsminister Jens Spahn legt die „Eck- } \\
\text { punkte zum Sofortprogramm Kranken- und Altenpflege“ } \\
\text { vor. Mit Wirkung zum 1. Januar } 2019 \text { soll ein umfang- } \\
\text { reiches Förderprogramm für Pflegestellen auch im Kran- } \\
\text { kenhaus beginnen. Neben Personaluntergrenzen für alle } \\
\text { bettenführenden Abteilungen sollen die Krankenkassen } \\
\text { nicht nur jede zusätzliche „Pflegestelle am Bett“ vollstän- } \\
\text { dig finanzieren, sondern auch die Tarifsteigerungen } \\
\text { übernehmen, und dies rückwirkend für 2018. Über den } \\
\text { auf } 4 \text { Mrd. Euro aufgestockten Strukturfonds sollen dann } \\
\text { auch die Ausbildungsstätten und die Digitalisierung der } \\
\text { Krankenhäuser zusätzlich zu den bestehenden Aufgaben } \\
\text { bezahlt werden. }\end{array}$ \\
\hline 23. Mai 2018 & Politik & $\begin{array}{l}\text { Bundesländer haben } \\
\text { ihre Investitionsförder- } \\
\text { mittel für Krankenhäu- } \\
\text { ser seit } 1991 \text { um fast } \\
50 \text { Prozent reduziert }\end{array}$ & $\begin{array}{l}\text { Was bisher immer nur vermutet wurde, bestätigt die } \\
\text { Bundesregierung in einer Antwort (Bundestags-Drs. } \\
\text { 19/2271) auf eine Kleine Anfrage der Fraktion Bündnis } \\
\text { 90/Die Grünen. Die Bundesländer haben ihre Investiti- } \\
\text { onsfördermittel für Krankenhäuser seit } 1991 \text { drastisch } \\
\text { reduziert. Der Anteil der Fördermittel an den Gesamt- } \\
\text { kosten der Krankenhäuser sank von 9,7 Prozent im Jahr } \\
1991 \text { auf nur noch 3,3 Prozent im Jahr } 2015 .\end{array}$ \\
\hline 8. bis 11. Mai 2018 & $\begin{array}{l}\text { Selbstver- } \\
\text { waltung }\end{array}$ & $\begin{array}{l}\text { Ärztetag fordert } 6.250 \\
\text { zusätzliche Medizin- } \\
\text { studienplätze }\end{array}$ & $\begin{array}{l}\text { Die Delegierten des Ärztetages fordern die Landesregie- } \\
\text { rungen dazu auf, die Zahl der Studienplätze von derzeit } \\
10.750 \text { Plätzen auf } 17.000 \text { jährlich zu erhöhen. Dies } \\
\text { entspricht der Anzahl von Medizinstudienplätzen vor der } \\
\text { Wiedervereinigung. }\end{array}$ \\
\hline 7. Mai 2018 & Politik & $\begin{array}{l}\text { BMG: Nur 2,3 Prozent } \\
\text { der Bevölkerung } \\
\text { werden künftig mehr } \\
\text { als } 30 \text { Minuten bis zu } \\
\text { einer Rettungsstelle } \\
\text { eines Krankenhauses } \\
\text { fahren müssen }\end{array}$ & $\begin{array}{l}\text { Laut Antwort des BMG auf eine parlamentarische An- } \\
\text { frage von Dr. Manuela Rottmann (Bündnis 90/Die } \\
\text { Grünen) zu den Auswirkungen des G-BA-Beschlusses } \\
\text { "stationäre Notfallversorgung“ ( } \$ 136 c \text { Absatz } 4 \text { SGB V) } \\
\text { wird darauf hingewiesen, dass nach der erfolgten Aus- } \\
\text { wirkungsanalyse nur 2,3 Prozent der Bevölkerung mehr } \\
\text { als } 30 \text { Minuten bis zu einer Rettungsstelle an einem } \\
\text { Krankenhaus fahren werden müssen. Hieraus ergibt sich } \\
\text { laut BMG zudem, dass in vielen der Bereiche, in denen } \\
\text { Fahrzeiten von mehr als } 30 \text { Minuten ermittelt wurden, } \\
\text { auch unter Status-quo-Bedingungen kein entspre- } \\
\text { chendes Krankenhaus vorhanden ist. }\end{array}$ \\
\hline
\end{tabular}


Krankenhauspolitische Chronik

\begin{tabular}{|c|c|c|c|}
\hline Termin & Leitbegriff & Vorgang & Legende \\
\hline 5. Mai 2018 & Politik & $\begin{array}{l}\text { Marburger Bund: } \\
\text { Krankenhausver- } \\
\text { sorgung braucht } \\
\text { Steuerung - Länder } \\
\text { in der Pflicht }\end{array}$ & $\begin{array}{l}\text { Der 1. Vorsitzende des Marburger Bundes (MB) und } \\
\text { CDU-MdB Rudolf Henke hat zum Auftakt der MB-Haupt- } \\
\text { versammlung in Erfurt an die Bundesregierung und die } \\
\text { Bundesländer appelliert, die Krankenhausstrukturen } \\
\text { zukunfts- und versorgungsorientiert weiterzuentwickeln. } \\
\text { Zudem seien, um eine gute stationäre Versorgung si- } \\
\text { cherzustellen, deutlich erhöhte Investitionen in Kranken- } \\
\text { häuser für Umstrukturierungen, neue Technologien und } \\
\text { Digitalisierung notwendig. }\end{array}$ \\
\hline 4. Mai 2018 & $\begin{array}{l}\text { Wissen- } \\
\text { schaft }\end{array}$ & $\begin{array}{l}\text { Intensivstationen sind } \\
\text { die Abteilungen mit } \\
\text { den meisten nosoko- } \\
\text { mialen Infektionen }\end{array}$ & $\begin{array}{l}\text { Nach einer europaweiten Auswertung des „European } \\
\text { Centre for Disease Prevention and Control“ (ECDC) in } \\
\text { Europa kann bei 8,4 Prozent aller Patienten auf einer } \\
\text { Intensivstation nach mehr als zwei Tagen Aufenthalt eine } \\
\text { nosokomiale Infektionen nachgewiesen werden (Annual } \\
\text { epidemiological report for 2016). }\end{array}$ \\
\hline 4. Mai 2018 & Politik & $\begin{array}{l}\text { Verbändeanhörung } \\
\text { zum Referentenent- } \\
\text { wurf einer Ausbil- } \\
\text { dungs- und Prüfungs- } \\
\text { verordnung für die } \\
\text { Pflegeberufe }\end{array}$ & $\begin{array}{l}\text { BMG und das Bundesministerium für Familie, Senioren, } \\
\text { Frauen und Jugend (BMFSFJ) haben die Verbändean- } \\
\text { hörung zum Referentenentwurf einer Ausbildungs- und } \\
\text { Prüfungsverordnung für die Pflegeberufe (Pflegeberufe- } \\
\text { Ausbildungs- und Prüfungsverordnung - PfIAPrV) durch- } \\
\text { geführt. Der Verordnungsentwurf regelt das Weitere zu } \\
\text { den Mindestanforderungen an die berufliche Pflegeaus- } \\
\text { bildung einschließlich der nach zwei Jahren zu absolvie- } \\
\text { renden Zwischenprüfung, die zu vermittelnden Kompe- } \\
\text { tenzen und das Verfahren der staatlichen Prüfungen. }\end{array}$ \\
\hline 30. April 2018 & Politik & $\begin{array}{l}\text { Richtlinie zu MDK- } \\
\text { Qualitätskontrollen in } \\
\text { Krankenhäusern tritt } \\
\text { unter Auflage in Kraft }\end{array}$ & $\begin{array}{l}\text { Das BMG sieht keine Rechtsgrundlage für eine generelle } \\
\text { Übermittlung des Kontrollberichts des MDKs an die } \\
\text { gesetzlichen Krankenkassen eines Landes. Daher ist } \\
\text { diese Regelungsvorgabe zu streichen. }\end{array}$ \\
\hline 25. April 2018 & $\begin{array}{l}\text { Rechtspre- } \\
\text { chung }\end{array}$ & $\begin{array}{l}\text { Einigungsstelle darf } \\
\text { keine Vorgaben } \\
\text { zur personellen } \\
\text { Mindestbesetzung } \\
\text { beschließen }\end{array}$ & $\begin{array}{l}\text { Der Betriebsrat eines Krankenhauses kann bei der Perso- } \\
\text { nalplanung des Arbeitgebers nicht erzwingbar mitbe- } \\
\text { stimmen, so das Landesarbeitsgericht Schleswig-Holstein } \\
\text { (Az.: } 6 \text { TaBV 21/17). Somit kann eine Einigungsstelle auch } \\
\text { aus Gründen der Mitbestimmung beim Gesundheits- } \\
\text { schutz gemäß § } 87 \text { Abs. } 1 \text { Nr. } 7 \text { BetrVG keine Vorgaben } \\
\text { über die personelle Mindestbesetzung beschließen. }\end{array}$ \\
\hline 24. April 2018 & $\begin{array}{l}\text { Rechtspre- } \\
\text { chung }\end{array}$ & $\begin{array}{l}\text { BSG schränkt Experi- } \\
\text { mentierklausel für } \\
\text { Krankenhausbehand- } \\
\text { lungen weitgehend } \\
\text { ein }\end{array}$ & $\begin{array}{l}\text { Das BSG entschied (Az.: B } 1 \text { KR 10/17 R (Erprobungsver- } \\
\text { fahren) und B } 1 \text { KR 10/17 R (Leiturteil)), dass die GKV eine } \\
\text { stationäre Liposuktion, auch wenn diese „das Potenzial } \\
\text { einer Behandlungsalternative“ habe, in der Regel nicht } \\
\text { vergüten muss. Das Qualitätsgebot sichere nicht nur die } \\
\text { Wirtschaftlichkeit, sondern auch die Gleichbehandlung } \\
\text { in der GKV und gewährleiste, „dass eine nicht ausrei- } \\
\text { chend erprobte Methode nicht zu Lasten der Kranken- } \\
\text { kassen erbracht werden darf“. }\end{array}$ \\
\hline 19. April 2018 & $\begin{array}{l}\text { Selbstver- } \\
\text { waltung }\end{array}$ & $\begin{array}{l}\text { Zukünftig auch Sicher- } \\
\text { stellungszuschläge für } \\
\text { Geburtshilfe möglich }\end{array}$ & $\begin{array}{l}\text { Die Geburtshilfe zählt im Sinne der Sicherstellungsrege- } \\
\text { lungen zukünftig zu den basisversorgungsrelevanten } \\
\text { Leistungen eines Krankenhauses. Zuschlagsfähig ist in } \\
\text { diesem Fall dann zudem die Vorhaltung einer Fachabtei- } \\
\text { lung Kinder- und Jugendmedizin. Dies hat der G-BA } \\
\text { beschlossen und in einer Richtlinie festgesetzt. }\end{array}$ \\
\hline
\end{tabular}


Krankenhauspolitische Chronik

\begin{tabular}{|c|c|c|c|}
\hline Termin & Leitbegriff & Vorgang & Legende \\
\hline 19. April 2018 & $\begin{array}{l}\text { Selbstver- } \\
\text { waltung }\end{array}$ & $\begin{array}{l}\text { Reformkonzept zur } \\
\text { stationären Notfallver- } \\
\text { sorgung beschlossen }\end{array}$ & $\begin{array}{l}\text { Nach zweijährigen Beratungen beschließt der G-BA } \\
\text { gegen das Votum der DKG die Reform der stationären } \\
\text { Notfallversorgung. Statt wie bisher } 1.748 \text { werden zukünf- } \\
\text { tig } 1.120 \text { Krankenhäuser die Notfallversorgung gemäß } \\
\text { eines „Stufenplans“ entweder als „Basis“- oder „Spitzen“- } \\
\text { Versorger gewährleisten. Details über die „Zuschlags- } \\
\text { vergütung" für diese Kliniken müssen GKV und DKG } \\
\text { noch verhandeln. In Kraft treten soll diese Vorgabe zum } \\
\text { 1. Januar } 2019 \text {. }\end{array}$ \\
\hline 19. April 2018 & $\begin{array}{l}\text { Wissen- } \\
\text { schaft }\end{array}$ & $\begin{array}{l}\text { RWI-Gutachten zur } \\
\text { Notfallversorgung in } \\
\text { Deutschland }\end{array}$ & $\begin{array}{l}\text { Es reichen bundesweit } 736 \text { Notfallzentren aus, um die } \\
\text { Bevölkerung im Notfall optimal zu versorgen. Das ergab } \\
\text { ein Gutachten des RWI - Leibnitz-Instituts für Wirt- } \\
\text { schaftsforschung, das im Auftrag der KBV erstellt wurde. }\end{array}$ \\
\hline 18. April 2018 & Politik & $\begin{array}{l}\text { Anhörung zum Pflege- } \\
\text { personal in Altenhei- } \\
\text { men und Krankenhäu- } \\
\text { sern }\end{array}$ & $\begin{array}{l}\text { Mit dem Pflegepersonal in Altenheimen und Kranken- } \\
\text { häusern befasst sich der AfG in einer öffentlichen Anhö- } \\
\text { rung. Dazu liegen den Sachverständigen vier Anträge } \\
\text { vor, zwei von der Linken und zwei von Bündnis 90/Die } \\
\text { Grünen. An dieser Anhörung nimmt unter anderen auch } \\
\text { Pflege-Azubi Alexander Jorde als Sachverständiger teil, } \\
\text { der das Thema im Bundestagswahlkampf publik ge- } \\
\text { macht hatte. }\end{array}$ \\
\hline 17. April 2018 & Politik & $\begin{array}{l}\text { Bundesgesundheits- } \\
\text { minister ermahnt die } \\
\text { Selbstverwaltungs- } \\
\text { partner }\end{array}$ & $\begin{array}{l}\text { Während des Frühlingsempfangs der DKG in Berlin } \\
\text { fordert Bundesgesundheitsminister Jens Spahn DKG und } \\
\text { GKV-Spitzenverband dazu auf, die Einführung von Perso- } \\
\text { naluntergrenzen bis Juni fristgerecht umzusetzen. „Ent- } \\
\text { scheiden Sie lieber selbst, bevor wir das im Ministerium } \\
\text { entscheiden“ schreibt er der gemeinsamen Selbstverwal- } \\
\text { tung ins Stammbuch. }\end{array}$ \\
\hline 17. April 2018 & $\begin{array}{l}\text { Selbstver- } \\
\text { waltung }\end{array}$ & $\begin{array}{l}\text { 7,5 Prozent Lohnstei- } \\
\text { gerungen für Beschäf- } \\
\text { tigte im Krankenhaus }\end{array}$ & $\begin{array}{l}\text { Der Tarifabschluss für den öffentlichen Dienst führt zu } \\
\text { finanziellen Verbesserungen für die Beschäftigten in den } \\
\text { Krankenhäusern. Sie werden u. a. künftig den vollen } \\
\text { Nachtzuschlag und in drei Stufen mehr Urlaub für die } \\
\text { Arbeit in der Wechselschicht erhalten. }\end{array}$ \\
\hline 16. April 2018 & Politik & $\begin{array}{l}\text { SPD für Selbstkosten- } \\
\text { deckungsprinzip und } \\
\text { gegen Zweckentfrem- } \\
\text { dung }\end{array}$ & $\begin{array}{l}\text { Die gesundheitspolitische Sprecherin der SPD-Bundes- } \\
\text { tagsfraktion, Sabine Dittmar, ist gegen gesonderte } \\
\text { Fallpauschalen für die Pflege, sogenannte Nursing Rela- } \\
\text { ted Groups (NRG), und plädiert stattdessen für die Ein- } \\
\text { führung eines Selbstkostenerstattungsprinzips zur } \\
\text { gesonderten Finanzierung der Pflege im Krankenhaus. } \\
\text { In diesem Zusammenhang warf sie den Krankenhäusern } \\
\text { vor, jährlich drei Milliarden Euro aus den DRGs, die für } \\
\text { Pflege vorgesehen seien, für andere Dinge wie Investit- } \\
\text { ionen zu verwenden. }\end{array}$ \\
\hline 11. April 2018 & Politik & $\begin{array}{l}\text { Neuer Patienten- } \\
\text { beauftragter der } \\
\text { Bundesregierung }\end{array}$ & $\begin{array}{l}\text { Der CDU-Bundestagsabgeordnete Dr. Ralf Brauksiepe } \\
\text { aus Nordrhein-Westfalen (NRW) wird der neue Patienten- } \\
\text { beauftragte der Bundesregierung. Damit ist das Bundes- } \\
\text { kabinett dem Vorschlag des Bundesministers für Ge- } \\
\text { sundheit Jens Spahn (CDU) gefolgt. }\end{array}$ \\
\hline
\end{tabular}


Krankenhauspolitische Chronik

\begin{tabular}{|c|c|c|c|}
\hline Termin & Leitbegriff & Vorgang & Legende \\
\hline 4. April 2018 & Qualität & $\begin{array}{l}\text { Jeder dritte Behand- } \\
\text { lungsfehler in Kliniken }\end{array}$ & $\begin{array}{l}\text { Im Jahr } 2017 \text { entfielen laut Behandlungsfehlerstatistik der } \\
\text { BÄK von den } 8.385 \text { Begutachtungsanträgen } 6.331 \text { auf } \\
\text { den Krankenhausbereich. Unfallchirurgie und Orthopädie } \\
\text { sind dabei mit 2.108 Prüfungen am häufigsten betroffen. }\end{array}$ \\
\hline 3. April 2018 & $\begin{array}{l}\text { Selbstver- } \\
\text { waltung }\end{array}$ & $\begin{array}{l}\text { InEK veröffentlicht } \\
\text { Katalog zur Bewer- } \\
\text { tung des Pflegeauf- } \\
\text { wands }\end{array}$ & $\begin{array}{l}\text { Das InEK hat ein Instrument zur Bewertung des Pflege- } \\
\text { aufwands in Krankenhäusern entwickelt. Der sog. Pflege- } \\
\text { last-Katalog weist für alle vollstationären Fallpauschalen } \\
\text { des DRG-Katalogs für } 2018 \text { ein tagesbezogenes Relativ- } \\
\text { gewicht für den Pflegeaufwand eines Patienten sowie } \\
\text { fallbezogene Relativgewichte für relevante Zusatzent- } \\
\text { gelte aus. Dieser Katalog soll bei den anstehenden Ver- } \\
\text { handlungen über Personaluntergrenzen auf betten- } \\
\text { führenden Stationen zur Ermittlung des Personalbedarfs } \\
\text { in der Pflege beitragen. }\end{array}$ \\
\hline 28. März 2018 & $\begin{array}{l}\text { Selbstver- } \\
\text { waltung }\end{array}$ & $\begin{array}{l}\text { InEK veröffentlicht } \\
\text { vierten Extremkosten- } \\
\text { bericht }\end{array}$ & $\begin{array}{l}\text { In dem Bericht wird dargestellt, dass die Kostenunterde- } \\
\text { ckungen der sogenannten Kostenausreißer durch ent- } \\
\text { sprechende Kostenüberdeckungen mehr als kompensiert } \\
\text { werden. Nach wie vor werden allerdings dem Bericht } \\
\text { zufolge atypische Krankheitsverläufe in Universitätskli- } \\
\text { niken und Maximalversorgern im Bereich der Regelleis- } \\
\text { tungen nicht hinreichend vergütet. }\end{array}$ \\
\hline 28. März 2018 & $\begin{array}{l}\text { Selbstver- } \\
\text { waltung }\end{array}$ & $\begin{array}{l}\text { Katalog der Investiti- } \\
\text { onsbewertungsrelati- } \\
\text { onen für das Jahr } 2018 \\
\text { veröffentlicht }\end{array}$ & $\begin{array}{l}\text { GKV-SV, PKV-Verband und DKG haben sich auf den } \\
\text { Katalog von Investitionsbewertungsrelationen für das } \\
\text { Jahr } 2018 \text { geeinigt. Für den PSY-Entgeltbereich war die } \\
\text { Berechnung von Investitionsbewertungsrelationen } \\
\text { aufgrund einer nicht ausreichenden Datenbasis bislang } \\
\text { nicht möglich. Die empirisch erhobenen Zahlen belegen } \\
\text { - wie auch in den vergangenen Jahren -, dass der be- } \\
\text { standserhaltende Investitionsbedarf der Krankenhäuser } \\
\text { bundesweit bei sechs Milliarden Euro liegt. Die Nutzung } \\
\text { des Instruments liegt in der Entscheidung des jeweiligen } \\
\text { Bundeslandes. Bisher werden die Investitionsbewer- } \\
\text { tungsrelationen in Berlin und Hessen angewendet. }\end{array}$ \\
\hline 21. März 2018 & Politik & $\begin{array}{l}\text { Neuer Pflegebevoll- } \\
\text { mächtigter der Bun- } \\
\text { desregierung bestellt }\end{array}$ & $\begin{array}{l}\text { Das Bundeskabinett hat Andreas Westerfellhaus auf } \\
\text { Vorschlag von Bundesgesundheitsminister Jens Spahn } \\
\text { zum neuen Bevollmächtigten der Bundesregierung für } \\
\text { Pflege bestellt. Er wird seine Tätigkeit voraussichtlich } \\
\text { Mitte April } 2018 \text { aufnehmen. }\end{array}$ \\
\hline 19. März 2018 & $\begin{array}{l}\text { Wissen- } \\
\text { schaft }\end{array}$ & $\begin{array}{l}\text { AOK-Bundesverband } \\
\text { und WIdO veröffentli- } \\
\text { chen Krankenhaus- } \\
\text { Report } 2018\end{array}$ & $\begin{array}{l}\text { Die Krankenhausstrukturen in Deutschland müssen } \\
\text { zentralisiert werden - diese Botschaft untermauern das } \\
\text { Wissenschaftliche Institut der AOK (WIdO) und der AOK- } \\
\text { Bundesverband bei ihrer Pressekonferenz zur Vorstellung } \\
\text { des Krankenhaus-Reports 2018. Im Blickpunkt der aktu- } \\
\text { ellen Ausgabe mit dem Schwerpunkt „Bedarf und Be- } \\
\text { darfsgerechtigkeit" steht die bedarfsgerechte Kranken- } \\
\text { hausplanung. }\end{array}$ \\
\hline 14. März 2018 & Politik & $\begin{array}{l}\text { Jens Spahn ist neuer } \\
\text { Bundesminister für } \\
\text { Gesundheit }\end{array}$ & $\begin{array}{l}\text { Bundespräsident Frank-Walter Steinmeier hat den bishe- } \\
\text { rigen Parlamentarischen Finanzstaatssekretär Jens } \\
\text { Spahn (CDU) zum Bundesminister für Gesundheit er- } \\
\text { nannt. Im Anschluss wurde Spahn im Deutschen Bundes- } \\
\text { tag vereidigt. Er folgt auf Hermann Gröhe (CDU), der das } \\
\text { Ministeramt seit Dezember } 2013 \text { ausgeübt hat. }\end{array}$ \\
\hline
\end{tabular}


Krankenhauspolitische Chronik

\begin{tabular}{|l|l|l|}
\hline Termin & Leitbegriff & Vorgang \\
\hline 13. März 2018 & $\begin{array}{l}\text { Wissen- } \\
\text { schaft }\end{array}$ & $\begin{array}{l}\text { Mehr Menschen we- } \\
\text { gen Depression in } \\
\text { Kliniken behandelt }\end{array}$ \\
\hline 2. März 2018 & Politik & $\begin{array}{l}\text { BMG veröffentlicht } \\
\text { vorläufige Finanz- } \\
\text { ergebnisse der } \\
\text { GKV 2017 }\end{array}$ \\
\hline 22. Februar 2018 & $\begin{array}{l}\text { Selbstver- } \\
\text { waltung }\end{array}$ & $\begin{array}{l}\text { Mindestmengenrege- } \\
\text { lung: Verzeichnis der } \\
\text { Landesverbände der }\end{array}$ \\
\hline 15. Februar 2018 & $\begin{array}{l}\text { Krankenkassen und } \\
\text { der Ersatzkassen zur } \\
\text { Übermittlung der }\end{array}$ \\
\hline Schaft & \begin{tabular}{l} 
Prognose liegt vor \\
\hline
\end{tabular} & $\begin{array}{l}\text { Erstmals mehr als eine } \\
\text { Milliarde Gesundheits- } \\
\text { ausgaben pro Tag }\end{array}$ \\
\hline
\end{tabular}

Legende

Die Zahl der Menschen in Deutschland, die wegen einer Depression in Krankenhäusern behandelt wurden, ist laut Destatis innerhalb von fünf Jahren um sieben Prozent gestiegen. Im Jahr 2016 wurden mehr als 263.000 Menschen vollstationär aufgenommen. Im Jahr 2011 waren es noch knapp 246.000 Patienten.

Die Ausgaben für Krankenhausbehandlung haben sich um 2,6 Prozent erhöht. Insgesamt erhielten die Krankenhäuser damit von den gesetzlichen Krankenkassen 2017 rund 1,9 Milliarden Euro höhere Finanzmittel als im entsprechenden Vorjahreszeitraum.

Die Mindestmengenregelung (Mm-R) sieht vor, dass der GKV-SV auf seiner Homepage ein verbindliches Verzeichnis der Kontaktadressen aller Landesverbände der Krankenkassen und der Ersatzkassen veröffentlicht, an welche - je Bundesland - die Prognosen der Krankenhäuser über die voraussichtliche Erfüllung der Mindestmengen zu übermitteln sind.

Die Gesundheitsausgaben in Deutschland haben laut Destatis im vergangenen Jahr erstmals die Marke von einer Milliarde Euro pro Tag überschritten. Für 2017 werden Ausgaben in Höhe von 374,2 Milliarden Euro prognostiziert. Das ist ein Anstieg um 4,9 Prozent im Vergleich zu 2016.

9. Februar $2018 \quad$ Politik

Antwort der Bundesregierung auf Kleine Anfrage der FDP-Fraktion

8. Februar 2018

Selbstverwaltung

\section{Februar 2018}

Politik

Selbstverwaltung

31. Januar 2018
Bundesvereinbarung zur Dokumentation der Leistungen der psychiatrischen Institutsambulanzen (PIA) geschlossen

Finale Fassung des Koalitionsvertrags liegt vor

Zwischenbericht zur Einführung von Pflegepersonaluntergrenzen

Gesundheitsausschuss neu konstituiert
Die Antwort der Bundesregierung (Drucksache 19/702) zur FDP-Anfrage (Drucksache 19/486) zur Situation der Krankenhäuser beinhaltet Aussagen zu Insolvenzen von Krankenhäusern sowie zur Investitionskosten-, Landesbasisfallwert- und Tarifentwicklung.

GKV-SV, PKV und DKG haben einen bundeseinheitlichen Katalog für die Dokumentation der Leistungen der psychiatrischen Institutsambulanzen (PIA-Doku-Vereinbarung) vereinbart, die zum 01.07.2018 in Kraft tritt. Der neue bundeseinheitliche Dokumentationskatalog umfasst nun 67 statt wie bisher vier Kodes. Er differenziert nach Art und Umfang der Leistung und enthält ergänzende Informationen über die medizinischen Inhalte der erbrachten Leistungen und das Behandlungssetting.

Nachdem die Koalitionsverhandlungen beendet wurden, liegt mit dem Titel „Ein neuer Aufbruch für Europa - Eine neue Dynamik für Deutschland - Ein neuer Zusammenhalt für unser Land: Koalitionsvertrag zwischen CDU, CSU und SPD" die finale Fassung des Koalitionsvertrags vor.

GKV-SV und DKG haben einen Zwischenbericht über den Stand der Umsetzung der gesetzlichen Aufgabe zur Identifikation pflegesensitiver Bereiche in der Krankenhausversorgung und der Festsetzung von Pflegepersonaluntergrenzen fristgerecht an das BMG übermittelt.

Der Gesundheitsausschuss des Bundestages hat sich mit seinen Mitgliedern neu konstituiert. 
Krankenhauspolitische Chronik

\begin{tabular}{|c|c|c|c|}
\hline Termin & Leitbegriff & Vorgang & Legende \\
\hline 30. Januar 2018 & Politik & $\begin{array}{l}\text { Gesundheitspolitische } \\
\text { Sprecher der Frakti- } \\
\text { onen im Deutschen } \\
\text { Bundestag }\end{array}$ & $\begin{array}{l}\text { Die Fraktionen im Deutschen Bundestag haben mit } \\
\text { Ausnahme der AfD ihre gesundheitspolitischen Sprecher } \\
\text { gewählt: } \\
\text { - Union: Karin Maag (zuständiger Fraktionsvize: Georg } \\
\text { Nüsslein) } \\
\text { - SPD: Sabine Dittmar (zuständiger Fraktionsvize: Karl } \\
\text { Lauterbach) } \\
\text { - FDP: Christine Aschenberg-Dugnus (zuständiger Frak- } \\
\text { tionsvize: Michael Theurer) } \\
\text { - Linke: Harald Weinberg (für Gesundheit zuständige } \\
\text { Fraktionsvize: Sabine Zimmermann) } \\
\text { - Grüne: Maria Klein-Schmeink (zuständige Arbeitskreis- } \\
\text { vorsitzende: Katja Dörner). } \\
\text { Als Vorsitzender des Gesundheitsausschusses wurde der } \\
\text { Pflegexperte der CDU Erwin Rüddel gewählt. }\end{array}$ \\
\hline 26. Januar 2018 & Politik & $\begin{array}{l}\text { Koalitionsverhand- } \\
\text { lungen starten }\end{array}$ & $\begin{array}{l}\text { Die Verhandlungen zur Großen Koalition aus Union und } \\
\text { SPD sind gestartet. Die Verhandlungsgruppen und der } \\
\text { Zeitplan liegen vor. }\end{array}$ \\
\hline 22. Januar 2018 & $\begin{array}{l}\text { Wissen- } \\
\text { schaft }\end{array}$ & $\begin{array}{l}\text { Kliniken melden } 2017 \\
\text { deutlich mehr Gewe- } \\
\text { bespender }\end{array}$ & $\begin{array}{l}\text { Deutsche Krankenhäuser vermitteln immer mehr Gewe- } \\
\text { bespenden von Verstorbenen für die Transplantation } \\
\text { etwa von Augenhornhäuten oder Herzklappen. Die Zahl } \\
\text { der eingegangenen Spendermeldungen ist innerhalb } \\
\text { der vergangenen drei Jahre von ungefähr } 27.000 \text { auf } \\
\text { über } 32.000 \text { gestiegen. Das ist ein Anstieg von mehr als } \\
20 \text { Prozent, so die Deutsche Gesellschaft für Gewebe- } \\
\text { transplantationen. }\end{array}$ \\
\hline 18. Januar 2018 & $\begin{array}{l}\text { Selbstver- } \\
\text { waltung }\end{array}$ & $\begin{array}{l}\text { Strukturierter Dialog: } \\
\text { Berichte und Weiter- } \\
\text { entwicklung }\end{array}$ & $\begin{array}{l}\text { Der G-BA hat zum Thema Strukturierter Dialog zwei } \\
\text { Beschlüsse getroffen: 1. Erstmals werden die jährlichen } \\
\text { Berichte zum Strukturierten Dialog mit den Krankenhäu- } \\
\text { sern durch den G-BA kommentiert. Diese Kommentie- } \\
\text { rung wird veröffentlicht. 2. Das IQTIG wurde mit einer } \\
\text { umfassenden Weiterentwicklung des Strukturierten } \\
\text { Dialogs mit den Krankenhäusern beauftragt. }\end{array}$ \\
\hline 17. Januar 2018 & Politik & $\begin{array}{l}\text { Antrag der Fraktion } \\
\text { Bündnis 90/Die Grü- } \\
\text { nen für ein Sofortpro- } \\
\text { gramm für mehr } \\
\text { Pflegepersonal im } \\
\text { Krankenhaus }\end{array}$ & $\begin{array}{l}\text { Die Grünen-Fraktion fordert in einem Antrag (Druck- } \\
\text { sache 19/447) im Bereich Pflege im Krankenhaus, ein } \\
\text { Sofortprogramm in Höhe von 1,3 Mrd. Euro im Jahr } \\
\text { aufzulegen, aus dessen Mitteln zusätzliche Pflegekräfte } \\
\text { zielgerichtet und verbunden mit einer Nachweispflicht } \\
\text { gefördert werden sowie verbindliche Personalbemes- } \\
\text { sungsinstrumente zu entwickeln, die sich am individu- } \\
\text { ellen Pflegebedarf der Patientinnen und Patienten orien- } \\
\text { tieren. }\end{array}$ \\
\hline
\end{tabular}


Krankenhauspolitische Chronik

\begin{tabular}{|c|c|c|c|}
\hline Termin & Leitbegriff & Vorgang & Legende \\
\hline 16. Januar 2018 & $\begin{array}{l}\text { Wissen- } \\
\text { schaft }\end{array}$ & $\begin{array}{l}\text { IAB-Studie zu Entgel- } \\
\text { ten von Pflegekräften }\end{array}$ & $\begin{array}{l}\text { Das Institut für Arbeitsmarkt und Berufsforschung der } \\
\text { Bundesagentur für Arbeit (IAB) hat eine Studie zu den } \\
\text { Entgelten von Pflegekräften erstellt. Danach sind seit } \\
\text { dem Jahr } 2012 \text { die Entgelte im Pflegebereich im Großen } \\
\text { und Ganzen entsprechend der allgemeinen Lohnent- } \\
\text { wicklung gestiegen. Bei Fachkräften in der Kranken- } \\
\text { pflege belief sich das Plus auf 8,9 Prozent. Fachkräfte in } \\
\text { der Krankenpflege verdienen mit durchschnittlich } \\
\text { 3.239 Euro geringfügig mehr als die Beschäftigten insge- } \\
\text { samt mit 3.133 Euro. Dabei ist zu berücksichtigen, dass in } \\
\text { der Krankenpflege häufig Zuschläge für spezielle Dienste } \\
\text { wie Nachtschichten oder Arbeit auf der Intensivstation } \\
\text { gezahlt werden. Dabei ist das Lohngefälle zwischen } \\
\text { Kranken- und Altenpflege weitgehend konstant geblie- } \\
\text { ben. Auch die regionalen Entgeltunterschiede sind nach } \\
\text { wie vor erheblich. }\end{array}$ \\
\hline 15. Januar 2018 & $\begin{array}{l}\text { Selbstver- } \\
\text { waltung }\end{array}$ & $\begin{array}{l}\text { Neue Pauschalen für } \\
\text { Organtransplanta- } \\
\text { tionen vereinbart }\end{array}$ & $\begin{array}{l}\text { Die Deutsche Stiftung Organtransplantation (DSO) hat } \\
\text { mit den Krankenkassen, der Bundesärztekammer und } \\
\text { den Kliniken die Pauschalen für das Jahr } 2018 \text { vereinbart. } \\
\text { Die Vertragspartner rechnen im laufenden Jahr mit } \\
\text { 2.830 Transplantationen und } 825 \text { Flügen für den Organ- } \\
\text { transport. Erstmals wird im Budget auch das Transplanta- } \\
\text { tionsregister berücksichtigt. }\end{array}$ \\
\hline 12. Januar 2018 & Politik & $\begin{array}{l}\text { Einigung in Sondie- } \\
\text { rungsgesprächen }\end{array}$ & $\begin{array}{l}\text { Union und SPD haben sich in den Sondierungsgesprä- } \\
\text { chen zur Vorbereitung einer Großen Koalition auf einen } \\
\text { 28-seitigen Text geeinigt. Die drei Parteivorsitzenden } \\
\text { Angela Merkel (CDU), Horst Seehofer (CSU) und Martin } \\
\text { Schulz (SPD) haben ihren Parteien die Aufnahme von } \\
\text { offiziellen Koalitionsverhandlungen empfohlen. Kran- } \\
\text { kenhauspolitisch relevante Punkte sind nachhaltige } \\
\text { Schritte zur Erreichung einer sektorenübergreifenden } \\
\text { Versorgung insbesondere bei der Notfallversorgung, } \\
\text { deutlich erhöhte Investitionen in Krankenhäuser für } \\
\text { Umstrukturierungen, neue Technologien und Digitalisie- } \\
\text { rung, sofort und spürbar verbesserte Arbeitsbedingun- } \\
\text { gen und Bezahlung in der Alten- und Krankenpflege } \\
\text { sowie die vollständige Refinanzierung von Tarifsteige- } \\
\text { rungen im Krankenhausbereich mit Nachweispflicht } \\
\text { sowie Pflege-Personaluntergrenzen für alle bettenfüh- } \\
\text { renden Abteilungen in Krankenhäusern. }\end{array}$ \\
\hline 28. Dezember 2017 & $\begin{array}{l}\text { Rechtspre- } \\
\text { chung }\end{array}$ & $\begin{array}{l}\text { BAG urteilt: Umkleide- } \\
\text { zeit ist Mehrarbeit }\end{array}$ & $\begin{array}{l}\text { Nach einem Urteil des Bundesarbeitsgerichts (BAG) ist } \\
\text { die für das An- und Ausziehen notwendige Umkleidezeit } \\
\text { des Klinikpersonals als Mehrarbeit zu vergüten, soweit } \\
\text { der jeweilige Tarifvertrag nichts Anderes vorsieht. In } \\
\text { seiner ständigen Rechtsprechung geht das BAG davon } \\
\text { aus, dass das An- und Ablegen „besonders auffälliger } \\
\text { Dienstkleidung“ vor Schichtbeginn zu vergütungspflich- } \\
\text { tiger Mehrarbeit führt. }\end{array}$ \\
\hline 21. Dezember 2017 & $\begin{array}{l}\text { Rechtspre- } \\
\text { chung }\end{array}$ & $\begin{array}{l}\text { Beschäftigungszeiten } \\
\text { in Privatkliniken gilt } \\
\text { auch bei Unikliniken } \\
\text { als ärztliche Tätigkeit }\end{array}$ & $\begin{array}{l}\text { Laut BAG, Az. } 6 \text { AZR 863/16, sind bei der Einstellung von } \\
\text { Ärzten an hessischen Universitätskliniken frühere ärzt- } \\
\text { liche Tätigkeiten, auch bei Privatkliniken, uneinge- } \\
\text { schränkt als Berufserfahrungszeiten zu berücksichtigen. }\end{array}$ \\
\hline
\end{tabular}


Krankenhauspolitische Chronik

\begin{tabular}{|c|c|c|c|}
\hline Termin & Leitbegriff & Vorgang & Legende \\
\hline 21. Dezember 2017 & $\begin{array}{l}\text { Selbstver- } \\
\text { waltung }\end{array}$ & $\begin{array}{l}\text { Qualitätskontrollen } \\
\text { des MDK in Kranken- } \\
\text { häusern }\end{array}$ & $\begin{array}{l}\text { Der G-BA beschließt die Erstfassung der Richtlinie zu } \\
\text { Grundsätzen der Qualitätskontrollen des MDK in Kran- } \\
\text { kenhäusern (§ 275a SGB V). Die Richtlinie, die erst nach } \\
\text { „Nichtbeanstandung“ durch das BMG in Kraft treten } \\
\text { wird, legt dabei auch die Voraussetzungen fest, aus } \\
\text { denen sich Qualitätskontrollen ergeben können, und } \\
\text { regelt generelle Fragen zu Beauftragung, Umfang, Art } \\
\text { und Verfahren der Kontrollen sowie zum Umgang mit } \\
\text { den Ergebnissen. }\end{array}$ \\
\hline 20. Dezember 2017 & $\begin{array}{l}\text { Selbstver- } \\
\text { waltung }\end{array}$ & $\begin{array}{l}\text { Eurotransplant-Budget } \\
\text { für } 2018 \text { vereinbart }\end{array}$ & $\begin{array}{l}\text { Die sogenannte Registrierungspauschale } 2018 \text {, die für } \\
\text { die Aufnahme eines Patienten auf die Warteliste von } \\
\text { Eurotransplant bezahlt wird, beträgt 1.166,- Euro. Für das } \\
\text { Jahr } 2018 \text { werden } 5.000 \text { Registrierungsfälle (2017: 5.400) } \\
\text { aus Deutschland erwartet. }\end{array}$ \\
\hline 19. Dezember 2017 & $\begin{array}{l}\text { Rechtspre- } \\
\text { chung }\end{array}$ & $\begin{array}{l}\text { Numerus clausus für } \\
\text { Medizin ist teilweise } \\
\text { verfassungswidrig } \\
\text { - Bund und Länder } \\
\text { müssen Auswahlkrite- } \\
\text { rien neu regeln }\end{array}$ & $\begin{array}{l}\text { Der Erste Senat am Bundesverfassungsgericht sieht die } \\
\text { Studienplatzvergabe für Medizin als teilweise verfas- } \\
\text { sungswidrig an. Zudem erkennt er im derzeitigen System } \\
\text { einen Verstoß gegen den Gleichheitsgrundsatz (Az. } 1 \\
\text { BvL 3/14, } 1 \text { BvL 4/14). Vor allem fordern die Verfassungs- } \\
\text { richter ein bundesweit standardisiertes System von } \\
\text { Eignungstests an Universitäten. Eine Neuregelung } \\
\text { soll daher bis zum 31. Dezember } 2019 \text { verabschiedet } \\
\text { werden. }\end{array}$ \\
\hline 15. Dezember 2017 & $\begin{array}{l}\text { Rechtspre- } \\
\text { chung }\end{array}$ & $\begin{array}{l}\text { Nur anwesend sein } \\
\text { reicht nicht, um "Chef- } \\
\text { arztbehandlung" } \\
\text { vergütet zu bekom- } \\
\text { men }\end{array}$ & $\begin{array}{l}\text { Das OLG Hamm hat entschieden (AZ. } 26 \text { U 74/17), dass } \\
\text { der Wahlarzt die seine Disziplin prägende Kernleistung } \\
\text { persönlich und eigenhändig erbringen muss, um eine } \\
\text { Vergütung aus einer Wahlleistungsvereinbarung geltend } \\
\text { machen zu können. Ist er zwar anwesend, führt die } \\
\text { Behandlung aber nicht eigenständig aus, so entsteht } \\
\text { kein Vergütungsanspruch. }\end{array}$ \\
\hline 6. Dezember 2017 & $\begin{array}{l}\text { Selbstver- } \\
\text { waltung }\end{array}$ & $\begin{array}{l}\text { Transplantationsre- } \\
\text { geln werden überwie- } \\
\text { gend von den Klinken } \\
\text { beachtet }\end{array}$ & $\begin{array}{l}\text { Die bei der Bundesärztekammer (BÄK) angesiedelte } \\
\text { Prüfungskommission und Überwachungskommission } \\
\text { zur Prüfung der Herz-, Lungen-, Leber-, Nieren- und } \\
\text { Pankreastransplantationsprogramme haben im Jahres- } \\
\text { bericht 2016/2017 keine Auffälligkeiten ergeben. Auch } \\
\text { die bereits abgeschlossenen Prüfungen bestätigen, dass } \\
\text { der ganz überwiegende Teil der Zentren ordnungsge- } \\
\text { mäß und korrekt arbeitet. Auffälligkeiten stellten die } \\
\text { Kommissionen lediglich in den Universitätskliniken } \\
\text { Berlin, Göttingen und Essen fest. }\end{array}$ \\
\hline 6. Dezember 2017 & Politik & $\begin{array}{l}\text { BMG veröffentlicht } \\
\text { Finanzergebnisse der } \\
\text { GKV im 1. bis 3. Quar- } \\
\text { tal } 2017\end{array}$ & $\begin{array}{l}\text { Die Ausgaben für Krankenhausbehandlung stiegen um } \\
\text { 2,4 Prozent. Insgesamt erhielten die Krankenhäuser } \\
\text { damit von den gesetzlichen Krankenkassen in den Mo- } \\
\text { naten Januar bis September rund 1,35 Milliarden Euro } \\
\text { höhere Finanzmittel als im entsprechenden Vorjahres- } \\
\text { zeitraum. Hinzu kommen bereits vereinbarte Preissteige- } \\
\text { rungen (Landesbasisfallwerte), die in diese Daten teils } \\
\text { noch nicht bzw. nicht für alle Monate einfließen konnten, } \\
\text { sowie weitere Verbesserungen im Krankenhausbereich. }\end{array}$ \\
\hline
\end{tabular}


Krankenhauspolitische Chronik

\begin{tabular}{|c|c|c|c|}
\hline Termin & Leitbegriff & Vorgang & Legende \\
\hline 5. Dezember 2017 & Qualität & $\begin{array}{l}\text { Chirurgische Fach- } \\
\text { gesellschaften fordern } \\
\text { höhere Mindestmen- } \\
\text { gen }\end{array}$ & $\begin{array}{l}\text { Die Deutsche Gesellschaft für Chirurgie (DGCH) und die } \\
\text { Deutsche Gesellschaft für Allgemein- und Viszeralchirur- } \\
\text { gie (DGAV) fordern höhere Mindestmengen als der G-BA. } \\
\text { Während der G-BA z. B. bei Bauchspeicheldrüsenkrebs } \\
\text { zehn Eingriffe pro Jahr vorgibt, werden seitens der } \\
\text { Chirurgen mindestens } 25 \text { Eingriffe gefordert. Zudem } \\
\text { bezeichnen die beiden chirurgischen Verbände die } \\
\text { gegenwärtige Regelung als „zahnlosen Tiger“ und } \\
\text { fordern deshalb, dass die Kassen finanziellen Druck auf } \\
\text { die Kliniken ausüben können sollen, die über Ausnah- } \\
\text { meregelungen versuchten, Mindestmengen zu unter- } \\
\text { schreiten. }\end{array}$ \\
\hline 5. Dezember 2017 & $\begin{array}{l}\text { Selbstver- } \\
\text { waltung }\end{array}$ & $\begin{array}{l}\text { Vereinbarung über die } \\
\text { Definition „Kranken- } \\
\text { hausstandort" }\end{array}$ & $\begin{array}{l}\text { DKG und GKV-SV verständigen sich über die Definition } \\
\text { von Klinikstandorten und auf die Einrichtung eines } \\
\text { bundesweiten Verzeichnisses. Die Definition wird für die } \\
\text { Qualitätssicherung, die Abrechnung, die Krankenhaus- } \\
\text { planung und für die Krankenhausstatistik benötigt. }\end{array}$ \\
\hline 29. November 2017 & $\begin{array}{l}\text { Wissen- } \\
\text { schaft }\end{array}$ & $\begin{array}{l}\text { Wenige Patienten - } \\
\text { hohes Risiko }\end{array}$ & $\begin{array}{l}\text { Laut Studie des Zentralinstituts für die kassenärztliche } \\
\text { Versorgung (Zi) werden im Durchschnitt rund 1,7 Pa- } \\
\text { tienten pro Stunde in der Notaufnahme eines Kranken- } \\
\text { hauses behandelt. Des Weiteren liegt die relative Über- } \\
\text { lebenswahrscheinlichkeit in größeren Notaufnahmen, } \\
\text { die eine durchschnittliche Auslastung von mehr als } \\
\text { 2,3 Patienten pro Stunde haben, je nach Indikation um } \\
30 \text { bis } 50 \text { Prozent höher als in unterdurchschnittlichen } \\
\text { Notfallambulanzen. }\end{array}$ \\
\hline 23. November 2017 & $\begin{array}{l}\text { Rechtspre- } \\
\text { chung }\end{array}$ & $\begin{array}{l}\text { Bademeister haften } \\
\text { wie Ärzte }\end{array}$ & $\begin{array}{l}\text { Bei schweren Pflichtverstößen kehrt sich bei Bademeis- } \\
\text { tern die Beweispflicht zu ihren Lasten um und sie haften } \\
\text { wie Ärzte, hat der Bundesgerichtshof entschieden } \\
\text { (Az.: III ZR 60/16). „Die Rechtslage ist in dieser Hinsicht } \\
\text { mit der im Arzthaftungsrecht vergleichbar. Hier wie dort } \\
\text { handelt es sich um Pflichten, die spezifisch auf den } \\
\text { Schutz von Leben und Gesundheit gerichtet sind“, erklär- } \\
\text { ten die Karlsruher Richter. Danach hat ein zum Unfallzeit- } \\
\text { punkt zwölfjähriges Mädchen noch Aussicht auf eine } \\
\text { Entschädigung. Sie hatte sich in einem Naturbad unter } \\
\text { Wasser im Seil einer Boje verfangen. Der Bademeister } \\
\text { hatte bemerkt, dass die Boje nicht mehr wie üblich aus } \\
\text { dem Wasser guckte, war aber nicht sofort ins Wasser } \\
\text { gesprungen. }\end{array}$ \\
\hline 20. November 2017 & Politik & $\begin{array}{l}\text { Koalitionsverhand- } \\
\text { lungen „Jamaika“ für } \\
\text { gescheitert erklärt }\end{array}$ & $\begin{array}{l}\text { Nach vier Wochen Sondierungsgesprächen und Verhand- } \\
\text { lungen scheitern die Koalitionsgespräche zwischen } \\
\text { Union, FDP und Bündnis 90/Die Grünen. }\end{array}$ \\
\hline
\end{tabular}


Krankenhauspolitische Chronik

\begin{tabular}{|c|c|c|c|}
\hline Termin & Leitbegriff & Vorgang & Legende \\
\hline 17. November 2017 & $\begin{array}{l}\text { Selbstver- } \\
\text { waltung }\end{array}$ & $\begin{array}{l}\text { Mindestmengenrege- } \\
\text { lungen für stationäre } \\
\text { Leistungen grundle- } \\
\text { gend überarbeitet }\end{array}$ & $\begin{array}{l}\text { Der G-BA hat eine Neufassung der Mindestmengenrege- } \\
\text { lungen für planbare stationäre Leistungen, bei denen } \\
\text { die Qualität des Behandlungsergebnisses von der Menge } \\
\text { der erbrachten Leistungen abhängig ist, sowie ein neues } \\
\text { Kapitel zu Mindestmengen in seiner Verfahrensordnung } \\
\text { beschlossen. Grund für die Änderungen war vor allem, } \\
\text { dass die Einführung von Mindestmengen bisher an den } \\
\text { Nachweis einer Abhängigkeit der Behandlungsqualität } \\
\text { von der erbrachten Leistungsmenge „in besonderem } \\
\text { Maße“ gebunden war. Die Unschärfe dieser Vorgabe } \\
\text { hatte zu zahlreichen Gerichtsprozessen geführt und die } \\
\text { Einführung von Mindestmengen faktisch lahmgelegt. }\end{array}$ \\
\hline 15. November 2017 & $\begin{array}{l}\text { Wissen- } \\
\text { schaft }\end{array}$ & $\begin{array}{l}\text { Studie: Politische } \\
\text { Einflussnahme führt in } \\
\text { kommunalen Kliniken } \\
\text { häufig zu einer } \\
\text { schlechteren wirt- } \\
\text { schaftlichen Lage }\end{array}$ & $\begin{array}{l}\text { Laut Studie der Wirtschaftsprüfungsgesellschaft BDO und } \\
\text { des Deutschen Krankenhausinstituts ist die wirtschaftliche } \\
\text { Lage vieler kommunaler Kliniken prekär. So habe fast ein } \\
\text { Drittel der Häuser von } 2014 \text { bis } 2016 \text { kontinuierlich Ver- } \\
\text { luste ausgewiesen. Dies sei „(...) auch auf die politische } \\
\text { Durchdringung der Aufsichtsräte zurückzuführen... } \\
\text { wonach in solchen Kliniken der Einfluss der Aufsichtsräte } \\
\text { auf die unternehmerischen Entscheidung stärker ausfalle“. }\end{array}$ \\
\hline 15. November 2017 & $\begin{array}{l}\text { Wissen- } \\
\text { schaft }\end{array}$ & $\begin{array}{l}\text { DKI: Wirtschaftliche } \\
\text { Situation verbessert - } \\
\text { drei von vier Kranken- } \\
\text { häusern } 2016 \text { mit } \\
\text { positivem Abschluss }\end{array}$ & $\begin{array}{l}\text { Nach Erkenntnis des „Krankenhaus-Barometers 2017“ } \\
\text { des Deutschen Krankenhausinstituts (DKI) erzielten } 2016 \\
\text { rund } 61 \text { Prozent der Krankenhäuser einen Jahresüber- } \\
\text { schuss, } 10 \text { Prozent ein ausgeglichenes Ergebnis. Nur } \\
\text { noch } 28 \text { Prozent mussten einen Verlust hinnehmen. Im } \\
\text { Vergleich zum Vorjahr - wonach } 58 \text { Prozent der Kliniken } \\
\text { Überschüsse aufwiesen, aber } 33 \text { Prozent Verluste verbu- } \\
\text { chen mussten - hat sich insgesamt die wirtschaftliche } \\
\text { Lage der Krankenhäuser verbessert. }\end{array}$ \\
\hline 10. November 2017 & $\begin{array}{l}\text { Wissen- } \\
\text { schaft }\end{array}$ & $\begin{array}{l}\text { Kosten für die statio- } \\
\text { näre Krankenhaus- } \\
\text { versorgung steigen } \\
\text { weiter }\end{array}$ & $\begin{array}{l}\text { Im Vergleich zu } 2015 \text { sind die Aufwendungen für die } \\
\text { stationäre Krankenhausversorgung } 2016 \text { laut Destatis } \\
\text { um 4,3 Prozent auf 87,8 Milliarden Euro angestiegen. } \\
\text { Umgerechnet auf die rund 19,5 Millionen Patienten, die } \\
2016 \text { vollstationär in Kliniken behandelt wurden, lagen } \\
\text { die Ausgaben je Fall bei durchschnittlich } 4.497 \text { Euro - } \\
\text { und damit 2,7 Prozent höher als im Jahr zuvor. }\end{array}$ \\
\hline 7. November 2017 & Politik & $\begin{array}{l}\text { Mehr Pflegepersonal } \\
\text { in Kliniken gefordert }\end{array}$ & $\begin{array}{l}\text { Die Linksfraktion fordert mit ihrem Antrag (19/30) die } \\
\text { Einführung einer verbindlichen Personalbemessung in } \\
\text { der Krankenhauspflege. Die von der großen Koalition } \\
\text { zuletzt beschlossenen Personaluntergrenzen drohten zu } \\
\text { scheitern, da die Beschäftigten und ihre Vertretungen } \\
\text { nicht mitentscheiden dürften. Stattdessen verhandelten } \\
\text { nur die Vertreter der Krankenhäuser mit denen der } \\
\text { Krankenversicherungen. }\end{array}$ \\
\hline 27. Oktober 2017 & $\begin{array}{l}\text { Selbstver- } \\
\text { waltung }\end{array}$ & $\begin{array}{l}\text { KBV kritisiert mangel- } \\
\text { hafte Umsetzung der } \\
\text { Abklärungspauschale } \\
\text { in der Notfallversor- } \\
\text { gung }\end{array}$ & $\begin{array}{l}\text { Nach Auffassung des stellvertretenden Vorstandsvorsit- } \\
\text { zenden der KBV, Dr. Stephan Hofmeister, wird die im } \\
\text { April eingeführte Abklärungspauschale in der Notfallver- } \\
\text { sorgung von den Krankenhäusern „,nach wie vor nicht } \\
\text { richtig verstanden." Dabei sei diese Pauschale „neues } \\
\text { Geld für eine Leistung, die erbracht wird“, erklärt er in } \\
\text { einem Videointerview mit KV-on. Die Pauschale beträgt } \\
\text { tagsüber 4,74 Euro und nachts 8,42 Euro. }\end{array}$ \\
\hline
\end{tabular}


Krankenhauspolitische Chronik

\begin{tabular}{|c|c|c|c|}
\hline Termin & Leitbegriff & Vorgang & Legende \\
\hline 26. Oktober 2017 & $\begin{array}{l}\text { Selbstver- } \\
\text { waltung }\end{array}$ & $\begin{array}{l}\text { DKG veröffentlicht } \\
\text { ihre Forderungen an } \\
\text { „Jamaika" }\end{array}$ & $\begin{array}{l}\text { Die DKG fordert, dass in den bereits laufenden schwarz- } \\
\text { gelb-grünen Sondierungsgesprächen bereits Verein- } \\
\text { barungen getroffen werden, um „nachhaltige Verbesse- } \\
\text { rungen der finanziellen und strukturellen Rahmenbedin- } \\
\text { gungen für die Krankenhäuser sicherzustellen“. }\end{array}$ \\
\hline 23. Oktober 2017 & $\begin{array}{l}\text { Wissen- } \\
\text { schaft }\end{array}$ & $\begin{array}{l}\text { Mehr als jeder dritte } \\
\text { Patient wurde operiert }\end{array}$ & $\begin{array}{l}\text { Laut Auskunft von Destatis wurde bei } 38 \text { Prozent } \\
\text { (7,1 Millionen) der knapp 19,0 Millionen stationär in all- } \\
\text { gemeinen Krankenhäusern behandelten Patientinnen und } \\
\text { Patienten eine Operation durchgeführt. Gegenüber dem } \\
\text { Vorjahr hat sich der Anteil nicht verändert. Des Weiteren } \\
\text { war gut die Hälfte der Behandelten, die sich } 2016 \text { wäh- } \\
\text { rend ihres Krankenhausaufenthaltes einem chirurgischen } \\
\text { Eingriff unterziehen mussten, } 60 \text { Jahre und älter. }\end{array}$ \\
\hline 20. Oktober 2017 & $\begin{array}{l}\text { Selbstver- } \\
\text { waltung }\end{array}$ & $\begin{array}{l}\text { Mehr Geld für Pflege } \\
\text { im Krankenhaus - Fall- } \\
\text { pauschalenkatalog } \\
2018 \text { vereinbart }\end{array}$ & $\begin{array}{l}\text { Die Selbstverwaltungspartner verständigen sich auf den } \\
\text { Fallpauschalenkatalog 2018, der die verbindliche Grund- } \\
\text { lage für die Abrechnungen zwischen Krankenversiche- } \\
\text { rern und Kliniken für stationäre somatische Leistungen } \\
\text { ist. Durch den neuen Katalog können die Krankenhäuser } \\
\text { u. a. für Patienten mit den höchsten Pflegegraden künf- } \\
\text { tig Zusatzentgelte abrechnen. Darüber hinaus haben die } \\
\text { Selbstverwaltungspartner auch den PEPP-Entgeltkatalog } \\
2018 \text { beschlossen und damit auch den finanziellen } \\
\text { Grundstein für die Behandlung von bestimmten psychi- } \\
\text { atrischen Patienten durch das multiprofessionelle } \\
\text { Krankenhausteam im häuslichen Umfeld gelegt (home } \\
\text { treatment oder Stationsäquivalente psychiatrische } \\
\text { Behandlung). }\end{array}$ \\
\hline 18. Oktober 2017 & Politik & $\begin{array}{l}\text { Jamaika-Sondie- } \\
\text { rungen starten }\end{array}$ & $\begin{array}{l}\text { Die Sondierungsgespräche über eine Jamaika-Koalition } \\
\text { starten zunächst mit Einzelgesprächen zwischen } \\
\text { einerseits Union, FDP und Grünen sowie andererseits } \\
\text { zwischen FDP und Grünen. Klar erkennbar ist, dass das } \\
\text { Thema Gesundheit - trotz Bürgerversicherungsproble- } \\
\text { matik - nicht das großes Streitthema sein wird. }\end{array}$ \\
\hline 16. Oktober 2017 & $\begin{array}{l}\text { Selbstver- } \\
\text { waltung }\end{array}$ & $\begin{array}{l}\text { Bundesbasisfallwert } \\
2018 \text { liegt bei } \\
3.467,30 \text { Euro }\end{array}$ & $\begin{array}{l}\text { Die Selbstverwaltungspartner auf Bundesebene ver- } \\
\text { ständigen sich auf den Bundesbasisfallwert für das } \\
\text { Jahr } 2018 \text { i. H. v. 3.467,30 Euro. Die obere Grenze des } \\
\text { Basisfallwertkorridors liegt damit bei 3.553,98 Euro } \\
\text { (plus 2,5 Prozent), die untere bei 3.431,93 Euro } \\
\text { (minus 1,02 Prozent). }\end{array}$ \\
\hline 4. Oktober 2017 & $\begin{array}{l}\text { Wissen- } \\
\text { schaft }\end{array}$ & $\begin{array}{l}\text { Weniger Kaiserschnitte } \\
\text { bei steigender } \\
\text { Geburtszahl }\end{array}$ & $\begin{array}{l}\text { Die Zahl der Krankenhausentbindungen in Deutschland } \\
\text { hat sich laut Destatis im Jahr } 2016 \text { gegenüber dem } \\
\text { Vorjahr um 6,3 Prozent auf } 761.777 \text { erhöht. Des Weiteren } \\
\text { ist in den vergangenen fünf Jahren die Kaiserschnittrate } \\
\text { von 32,2 Prozent auf 30,5 Prozent gesunken. }\end{array}$ \\
\hline 4. Oktober 2017 & $\begin{array}{l}\text { Recht- } \\
\text { sprechung }\end{array}$ & $\begin{array}{l}\text { Auswahlverfahren } \\
\text { für Medizinstudium } \\
\text { auf dem Prüfstand }\end{array}$ & $\begin{array}{l}\text { Das Bundesverfassungsgericht stellt die Zulassungsbe- } \\
\text { dingungen für das Medizinstudium mit einer Konzentra- } \\
\text { tion auf die Abiturnote auf den Prüfstand. Im Mittelpunkt } \\
\text { der mündlichen Verhandlung steht die Frage, ob das } \\
\text { derzeitige Verfahren mit dem Grundrecht auf freie Wahl } \\
\text { des Berufs und des Ausbildungsplatzes sowie dem Gleich- } \\
\text { heitsgrundsatz vereinbar ist (Az. } 1 \text { BvL 3/14 und 4/14). }\end{array}$ \\
\hline
\end{tabular}


Krankenhauspolitische Chronik

\begin{tabular}{|c|c|c|c|}
\hline Termin & Leitbegriff & Vorgang & Legende \\
\hline 1. Oktober 2017 & $\begin{array}{l}\text { Gesetz- } \\
\text { gebung }\end{array}$ & $\begin{array}{l}\text { Verpflichtendes Ent- } \\
\text { lassmanagement }\end{array}$ & $\begin{array}{l}\text { Krankenhäuser sind seit } 1 \text {. Oktober } 2017 \text { verpflichtet, } \\
\text { ihren Patienten ein standardisiertes Entlassmanagement } \\
\text { anzubieten. }\end{array}$ \\
\hline $\begin{array}{l}\text { 29. September } \\
2017\end{array}$ & $\begin{array}{l}\text { Wissen- } \\
\text { schaft }\end{array}$ & $\begin{array}{l}\text { Destatis: Orientie- } \\
\text { rungswert } 2018 \text { be- } \\
\text { trägt 2,11 Prozent }\end{array}$ & $\begin{array}{l}\text { Der Orientierungswert } 2018 \text { für Krankenhäuser beträgt } \\
\text { 2,11 Prozent. Der Wert gibt die durchschnittliche jähr- } \\
\text { liche prozentuale Veränderung der Krankenhauskosten } \\
\text { wieder, die ausschließlich aus Preis- oder Verdienstände- } \\
\text { rungen resultiert. Die Personalkosten sind um 2,73 } \\
\text { Prozent und Sachkosten um 1,11 Prozent gestiegen. }\end{array}$ \\
\hline $\begin{array}{l}\text { 22. September } \\
2017\end{array}$ & $\begin{array}{l}\text { Selbstver- } \\
\text { waltung }\end{array}$ & $\begin{array}{l}\text { InEK erweitert Kalkula- } \\
\text { tionsstichprobe }\end{array}$ & $\begin{array}{l}\text { Das InEK hat } 80 \text { weitere Krankenhäuser ausgelost, die } \\
\text { ihre Daten zur Kalkulation von Fallpauschalen zur Verfü- } \\
\text { gung stellen sollen. Im Rahmen der zweiten Ziehung } \\
\text { wurden je } 20 \text { Kliniken für den Bereich „DRG“ und „PSY“ } \\
\text { zur Teilnahme an der Kostenerhebung bestimmt, weitere } \\
40 \text { Häuser wurden für Verbesserung der Investitionsbe- } \\
\text { wertungsrelationen ausgewählt. }\end{array}$ \\
\hline $\begin{array}{l}\text { 19. September } \\
2017\end{array}$ & $\begin{array}{l}\text { Selbstver- } \\
\text { waltung }\end{array}$ & $\begin{array}{l}\text { Krankenkassen kün- } \\
\text { digen Zentrenverein- } \\
\text { barung }\end{array}$ & $\begin{array}{l}\text { Der GKV-Spitzenverband (GKV-SV) hat gemeinsam mit } \\
\text { dem Verband der Privaten Krankenversicherung (PKV) } \\
\text { den Vertrag zur Finanzierung von Zentren inklusive } \\
\text { seiner Anlage gekündigt. Der GKV-SV beschreibt den } \\
\text { Schritt als „unvermeidbar", denn der Vertrag zur Zentren- } \\
\text { finanzierung basiere auf Festlegungen der Bundes- } \\
\text { schiedsstelle, die aus Kassensicht dem eigentlichen } \\
\text { Gesetzesauftrag aus dem Krankenhausstrukturgesetz } \\
\text { (KHSG) nicht gerecht würden. }\end{array}$ \\
\hline $\begin{array}{l}\text { 19. September } \\
2017\end{array}$ & $\begin{array}{l}\text { Selbstver- } \\
\text { waltung }\end{array}$ & $\begin{array}{l}\text { IQTIG legt Bericht zum } \\
\text { Strukturierten Dialog } \\
2016 \text { vor: } 714 \text { Kliniken } \\
\text { fielen auf }\end{array}$ & $\begin{array}{l}\text { Laut Bericht haben } 1.834 \text { Krankenhäuser für das Erfas- } \\
\text { sungsjahr } 2015 \text { ihre Daten für die externe Qualitätssiche- } \\
\text { rung übermittelt. Bei } 714 \text { Kliniken wurde aufgrund von } \\
\text { Auffälligkeiten in der Dokumentation ein Strukturierter } \\
\text { Dialog geführt. In } 372 \text { Fällen lagen tatsächlich Dokumen- } \\
\text { tationsfehler vor, } 219 \text { Datensätze waren unvollständig }\end{array}$ \\
\hline 8. September 2017 & Politik & $\begin{array}{l}\text { BMG veröffentlicht } \\
\text { Finanzergebnisse der } \\
\text { GKV im 1. Halbjahr } \\
2017\end{array}$ & $\begin{array}{l}\text { Die Ausgaben für Krankenhausbehandlung stiegen im } \\
\text { 1. Halbjahr } 2017 \text { absolut um 2,5 Prozent (je Versicherten } \\
\text { um 1,3 Prozent). Insgesamt erhielten die Krankenhäuser } \\
\text { damit von den gesetzlichen Krankenkassen in den } \\
\text { Monaten Januar bis Juni rund 0,94 Milliarden Euro } \\
\text { höhere Finanzmittel als im entsprechenden Vorjahres- } \\
\text { zeitraum. }\end{array}$ \\
\hline 7. September 2017 & Politik & $\begin{array}{l}\text { Fraktion der Grünen } \\
\text { stellen Zehn-Punkte- } \\
\text { Programm für eine } \\
\text { bessere Notfallversor- } \\
\text { gung vor }\end{array}$ & $\begin{array}{l}\text { Die AG Gesundheit der grünen Bundestagsfraktion hat } \\
\text { ein Positionspapier zur Notfallversorgung vorgelegt. Ziel } \\
\text { ist es, die Sektorengrenzen aufzubrechen und die Struk- } \\
\text { turen der Notfallversorgung so zu reformieren, dass sie } \\
\text { die Bedürfnisse der Patienten und Patientinnen treffen. }\end{array}$ \\
\hline
\end{tabular}


Krankenhauspolitische Chronik

\begin{tabular}{|c|c|c|c|}
\hline Termin & Leitbegriff & Vorgang & Legende \\
\hline 7. September 2017 & $\begin{array}{l}\text { Wissen- } \\
\text { schaft }\end{array}$ & $\begin{array}{l}\text { SVR-Gesundheit stellt } \\
\text { Reform der Notfallver- } \\
\text { sorgung vor }\end{array}$ & $\begin{array}{l}\text { Der Sachverständigenrat zur Begutachtung der Entwick- } \\
\text { lung im Gesundheitswesen (SVR-Gesundheit) hat im } \\
\text { Rahmen eines Werkstattgesprächs Analysen zum Status } \\
\text { quo sowie Empfehlungen zur Zukunft der Notfallversor- } \\
\text { gung in Deutschland vorgestellt. Der Rat empfiehlt die } \\
\text { Schaffung voll integrierter, regionaler Leitstellen, die } \\
\text { über eine bundeseinheitliche Rufnummer erreichbar } \\
\text { sind und je nach Patientenanliegen die individuell beste } \\
\text { Versorgungsoption wählen. Das für viele Patienten } \\
\text { unverständliche Nebeneinander verschiedener Rufnum- } \\
\text { mern (vor allem } 112 \text { und } 116117 \text { ) soll damit zukünftig } \\
\text { entfallen. Wichtig sind dem Rat die nahtlose Verzahnung } \\
\text { der bislang drei getrennten Bereiche und die damit } \\
\text { einhergehende Bildung von Zentren. Die neu geschaf- } \\
\text { fenen sektorenübergreifenden integrierten Notfallzen- } \\
\text { tren sollen an bestehenden Kliniken, aber als eigenstän- } \\
\text { dige organisatorisch-wirtschaftliche Einheit angesiedelt } \\
\text { werden. }\end{array}$ \\
\hline 6. September 2017 & $\begin{array}{l}\text { Wissen- } \\
\text { schaft }\end{array}$ & $\begin{array}{l}\text { AQUA-Institut legt } \\
\text { ein von Zi beauf- } \\
\text { tragtes Konzept zur } \\
\text { Notfallversorgung vor }\end{array}$ & $\begin{array}{l}\text { Das Zentralinstitut für die kassenärztliche Versorgung } \\
\text { (Zi) hat das AQUA-Institut beauftragt, ein Konzept zur } \\
\text { gezielten Steuerung von Notfallpatienten zu erstellen. } \\
\text { Der Projektbericht „Instrumente und Methoden zur } \\
\text { Ersteinschätzung von Notfallpatienten - Bestandsauf- } \\
\text { nahme und Konzeptentwicklung für die kassenärztliche } \\
\text { Notfallversorgung“ liegt nun vor. Zentrale Punkte sind } \\
\text { eine standardisierte Ersteinschätzung, wenn Patienten } \\
\text { in der Notaufnahme eines Krankenhauses erscheinen, } \\
\text { mit anschließender Weiterleitung in die stationäre oder } \\
\text { ambulante Versorgung, und idealerweise eine bereits im } \\
\text { Vorfeld eines Rettungsstellenbesuchs erfolgte Kontakt- } \\
\text { aufnahme mit der bundesweit geltenden Nummer für } \\
\text { den ärztlichen Bereitschaftsdienst unter } 116117 \text { durch } \\
\text { den Patienten zur Einschätzung und gezielten Versor- } \\
\text { gungssteuerung. }\end{array}$ \\
\hline 1. September 2017 & $\begin{array}{l}\text { Selbstver- } \\
\text { waltung }\end{array}$ & Einigung auf Tarifrate & $\begin{array}{l}\text { Nach intensiven Verhandlungen haben sich die Vertrags- } \\
\text { parteien auf Bundesebene auf eine pauschale Ver- } \\
\text { einbarung einer Tariferhöhungsrate } 2017 \text { verständigt. } \\
\text { Auf dieser Basis ergibt sich eine Erhöhungsrate von } \\
0,48 \text { Prozent und somit eine anteiligen Erhöhungsrate im } \\
\text { DRG-Bereich von 0,16 Prozent. }\end{array}$ \\
\hline 31. August 2017 & $\begin{array}{l}\text { Selbstver- } \\
\text { waltung }\end{array}$ & $\begin{array}{l}\text { Landesbasisfallwerte: } \\
\text { Klinikerlöse steigen } \\
\text { um vier Prozent }\end{array}$ & $\begin{array}{l}\text { Die Erlöse der somatischen Krankenhäuser aus den Fall- } \\
\text { pauschalen steigen } 2017 \text { auf 70,7 Milliarden Euro. Das sind } \\
\text { rund drei Milliarden Euro oder 4,1 Prozent mehr als im } \\
\text { Vorjahr. Diese Zahlen ergeben sich aus den diesjährigen } \\
\text { Landesbasisfallwertverhandlungen auf Landesebene. }\end{array}$ \\
\hline 29. August 2017 & Politik & $\begin{array}{l}\text { BMG: Grundlohnrate } \\
2018 \text { liegt bei } \\
\text { 2,97 Prozent }\end{array}$ & $\begin{array}{l}\text { Das BMG gab bekannt, dass die Grundlohnrate für das } \\
\text { Jahr } 2018 \text { 2,97 Prozent beträgt. Der Wert beziffert die } \\
\text { Veränderungsrate der beitragspflichtigen Einnahmen } \\
\text { aller Mitglieder der gesetzlichen Krankenkassen für das } \\
\text { Jahr } 2018 .\end{array}$ \\
\hline
\end{tabular}


Krankenhauspolitische Chronik

\begin{tabular}{|c|c|c|c|}
\hline Termin & Leitbegriff & Vorgang & Legende \\
\hline 24. August 2017 & $\begin{array}{l}\text { Selbstver- } \\
\text { waltung }\end{array}$ & $\begin{array}{l}\text { Qualitätssicherungs- } \\
\text { Richtlinie Früh- } \\
\text { und Reifgeborene } \\
\text { (QFR-RL): Beschluss } \\
\text { zu den Vorgaben } \\
\text { zur schichtbezogenen } \\
\text { Dokumentation und } \\
\text { Ausgestaltung des } \\
\text { klärenden Dialogs } \\
\text { treten in Kraft }\end{array}$ & $\begin{array}{l}\text { Die Beschlüsse des G-BA zur Änderung der Qualitäts- } \\
\text { sicherungs-Richtlinie Früh- und Reifgeborene (QFR-RL) } \\
\text { im Hinblick auf die Ausgestaltung der Schritte und } \\
\text { Maßnahmen, die greifen, wenn Perinatalzentren die } \\
\text { Personalvorgaben für die Intensivpflege von Frühgebo- } \\
\text { renen nicht erfüllen, sind im Bundesanzeiger veröffent- } \\
\text { licht. Damit ist die konkrete Ausgestaltung des Verfah- } \\
\text { rens verbindlich. Ergänzend sind nun auch die konkreten } \\
\text { Angaben zu einer schichtgenauen Dokumentation in } \\
\text { Form eines Musterformulars verpflichtend, die für den } \\
\text { Nachweis des geforderten Personalschlüssels erforder- } \\
\text { lich sind. }\end{array}$ \\
\hline 24. August 2017 & $\begin{array}{l}\text { Selbstver- } \\
\text { waltung }\end{array}$ & $\begin{array}{l}\text { Frühchen-Stationen: } \\
\text { Strukturabfrage zur } \\
\text { Personalausstattung } \\
\text { beschlossen }\end{array}$ & $\begin{array}{l}\text { Geburtskliniken, die schwerpunktmäßig Frühchen und } \\
\text { kranke Neugeborene versorgen, sollen künftig einmal im } \\
\text { Jahr über ihre Personalausstattung berichten. Eine ent- } \\
\text { sprechende Strukturabfrage hat jetzt der Gemeinsame } \\
\text { Bundesausschuss (GBA) beschlossen. Das Gremium will } \\
\text { auf diesem Weg in Erfahrung bringen, inwieweit die } \\
\text { Frühchen-Versorgung den Vorgaben zur Qualitätssiche- } \\
\text { rung entspricht. }\end{array}$ \\
\hline 18. August 2017 & $\begin{array}{l}\text { Selbstver- } \\
\text { waltung }\end{array}$ & $\begin{array}{l}\text { IGES-Befragung zur } \\
\text { Notfallversorgung } \\
\text { läuft länger }\end{array}$ & $\begin{array}{l}\text { Krankenhäuser, die sich an der Umfrage des IGES Insti- } \\
\text { tuts zu Notfallstrukturen beteiligen, haben nun bis zum } \\
\text { 31. August } 2017 \text { Zeit. Das hat der Gemeinsame Bundes- } \\
\text { ausschuss (GBA) mitgeteilt, der die Befragung in Auftrag } \\
\text { gegeben hat. Bei der Umfrage können die knapp } 2.000 \\
\text { zugelassenen Krankenhäuser eine Selbsteinschätzung } \\
\text { zum bisherigen G-BA-Konzept zu den gestuften Notfall- } \\
\text { strukturen abgeben. }\end{array}$ \\
\hline 14. August 2017 & $\begin{array}{l}\text { Wissen- } \\
\text { schaft }\end{array}$ & $\begin{array}{l}\text { Destatis: Mehr } \\
\text { Patienten verursachen } \\
\text { weniger Belegungs- } \\
\text { tage }\end{array}$ & $\begin{array}{l}\text { Rund 19,5 Millionen Patienten wurden } 2016 \text { vollstationär } \\
\text { im Krankenhaus behandelt. Im Vergleich zum Jahr } 2010 \\
\text { (18,0 Millionen) ist die Zahl der Behandelten um 8,3 } \\
\text { Prozent gestiegen. Während die Zahl der Patienten stieg, } \\
\text { sank ihre Aufenthaltsdauer. } 2016 \text { lag ein Kranker im } \\
\text { Durchschnitt 7,3 Tage im Krankenhaus. } 2010 \text { waren es } \\
\text { noch 7,9 Tage. Das gilt auch für Berechnungs- und Bele- } \\
\text { gungstage: Ihre Anzahl verringerte sich auf } 142 \text { Millionen } \\
\text { im Jahr } 2016 \text {. }\end{array}$ \\
\hline 10. August 2017 & $\begin{array}{l}\text { Wissen- } \\
\text { schaft }\end{array}$ & $\begin{array}{l}\text { Umfrage: } \\
\text { Gute Zeugnisse für } \\
\text { Geburtkliniken }\end{array}$ & $\begin{array}{l}83 \text { Prozent der Mütter empfehlen die Geburtsklinik } \\
\text { weiter, in der sie selbst entbunden haben. Das zeigt die } \\
\text { Auswertung einer Umfrage von AOK, BARMER und der } \\
\text { „Weissen Liste“. Fast } 90 \text { Prozent lobten den Umgang mit } \\
\text { den Neugeborenen. Nicht minder zufrieden sind die } \\
\text { Mütter mit der Arbeit der Hebammen. Allerdings zeigt } \\
\text { die Umfrage auch große Unterschiede zwischen einzel- } \\
\text { nen Kliniken. }\end{array}$ \\
\hline 10. August 2017 & $\begin{array}{l}\text { Wissen- } \\
\text { schaft }\end{array}$ & $\begin{array}{l}\text { Pflege: Freie Stellen } \\
\text { bleiben oft lange } \\
\text { unbesetzt }\end{array}$ & $\begin{array}{l}\text { Freie Stellen für examinierte Gesundheits- und Kranken- } \\
\text { pfleger bleiben ca. } 140 \text { Tage unbesetzt, im Vergleich zum } \\
\text { Vorjahr } 16 \text { Tage länger. Das zeigt eine aktuelle Analyse } \\
\text { der Bundesagentur für Arbeit. Die durchschnittliche } \\
\text { Vakanzzeit liegt } 39 \text { Prozent über dem Durchschnitt aller } \\
\text { Berufe. }\end{array}$ \\
\hline
\end{tabular}


Krankenhauspolitische Chronik

\begin{tabular}{|c|c|c|c|}
\hline Termin & Leitbegriff & Vorgang & Legende \\
\hline 2. August 2017 & Politik & $\begin{array}{l}\text { Krankenhausstruktur- } \\
\text { fonds: Bundesver- } \\
\text { sicherungsamt zieht } \\
\text { erste Bilanz }\end{array}$ & $\begin{array}{l}66 \text { Anträge für Mittel aus dem Strukturfonds sind zum } \\
\text { 31. Juli } 2017 \text { beim Bundesversicherungsamt (BVA) einge- } \\
\text { gangen. Der Fördertopf des BVA umfasst } 500 \text { Millionen } \\
\text { Euro. Mehr als die Hälfte der Projekte zielt auf die Kon- } \\
\text { zentration stationärer Versorgungskapazitäten. }\end{array}$ \\
\hline 1. August 2017 & $\begin{array}{l}\text { Selbstver- } \\
\text { waltung }\end{array}$ & $\begin{array}{l}\text { Vereinbarung zur } \\
\text { stationsäquivalenten } \\
\text { psychiatrischen Be- } \\
\text { handlung geschlossen } \\
\text { und in Kraft }\end{array}$ & $\begin{array}{l}\text { GKV-SV, PKV und DKG haben die Vereinbarung zur } \\
\text { stationsäquivalenten psychiatrischen Behandlung ge- } \\
\text { schlossen. Darin sind die Anforderungen an die Doku- } \\
\text { mentation, die Qualität der Leistungserbringung und die } \\
\text { Beauftragung Dritter definiert. Die Vereinbarung ist am } \\
\text { 1. August in Kraft getreten. }\end{array}$ \\
\hline 1. August 2017 & $\begin{array}{l}\text { Selbstver- } \\
\text { waltung }\end{array}$ & $\begin{array}{l}\text { InEK berichtet über die } \\
\text { Kalkulation der Investi- } \\
\text { tionsbewertungsrelati- } \\
\text { onen (IBR) } 2017\end{array}$ & $\begin{array}{l}\text { Die IBR für das Jahr } 2017 \text { beruhen auf den Daten von } \\
45 \text { Krankenhäusern, die freiwillig Investitionskosten } \\
\text { berechnet und dem InEK ihre Ergebnisse zur Verfügung } \\
\text { gestellt haben. }\end{array}$ \\
\hline 25. Juli 2017 & $\begin{array}{l}\text { Wissen- } \\
\text { schaft }\end{array}$ & $\begin{array}{l}\text { DKG-Gutachten zur } \\
\text { „Personalsituation auf } \\
\text { Intensivstationen“ }\end{array}$ & $\begin{array}{l}\text { Die DKG hat eine Analyse zur Personalsituation auf Inten- } \\
\text { sivstationen vorgestellt und kommt zu dem Schluss, dass } \\
\text { die Versorgung der Patienten objektiv gut sei. Laut } \\
\text { Gutachten lag das Verhältnis von Intensivpatienten zu } \\
\text { Pflegekräften } 2015 \text { im Durchschnitt bei 2,2 Fällen pro } \\
\text { Schicht und Pflegekraft (VK). Die Empfehlung der Fach- } \\
\text { gesellschaft Deutsche Interdisziplinäre Vereinigung für } \\
\text { Intensiv- und Notfallmedizin (DIVI) eines Pflegekraft-zu- } \\
\text { Patienten-Verhältnisses von zwei Fällen pro Schicht und } \\
\text { Pflegekraft wird im Mittel in etwa erreicht. Die repräsen- } \\
\text { tative schriftliche Befragung zur Personalsituation in } \\
\text { Intensivpflege und Intensivmedizin erfolgte von Sep- } \\
\text { tember bis November } 2016 \text { in } 1.261 \text { deutschen Kranken- } \\
\text { häusern, von denen ein Viertel den zwölfseitigen Frage- } \\
\text { bogen mit rund } 150 \text { Punkten zurückgeschickt hatte. }\end{array}$ \\
\hline 21. Juli 2017 & $\begin{array}{l}\text { Selbstver- } \\
\text { waltung }\end{array}$ & $\begin{array}{l}\text { Hygieneförderung: } \\
\text { Kliniken haben bis } \\
2016 \text { knapp } 225 \text { Milli- } \\
\text { onen Euro abgerufen }\end{array}$ & $\begin{array}{l}\text { Der GKV-SV hat am 30.06.2017 den Bericht über das } \\
\text { Hygienesonderprogramm } 2013 \text { bis } 2016 \text { an das Bundes- } \\
\text { ministerium für Gesundheit (BMG) übermittelt. Der Be- } \\
\text { richt belegt, dass die GKV den Krankenhäusern in diesem } \\
\text { Zeitraum zusätzliche Finanzmittel in Höhe von } \\
\text { ca. } 224,6 \text { Mio. Euro zur Verfügung gestellt hat. Bundesweit } \\
\text { verzeichnete der GKV-SV } 541 \text { Vereinbarungen zu Hygiene- } \\
\text { fachkräften und 1.282 zu hygienebeauftragten Ärzten. }\end{array}$ \\
\hline 19. Juli 2017 & Politik & $\begin{array}{l}\text { Mindestlöhne in der } \\
\text { Pflege steigen ab } \\
\text { Januar } 2018\end{array}$ & $\begin{array}{l}\text { Eine entsprechende Verordnung von Bundesarbeits- } \\
\text { ministerin Andrea Nahles (SPD) zur Erhöhung der Min- } \\
\text { destlöhne passierte am Mittwoch das Bundeskabinett. } \\
\text { Der Mindestlohn in der Pflege liegt dann bei 10,55 Euro } \\
\text { pro Stunde im Westen und 10,05 Euro im Osten Deutsch- } \\
\text { lands. In zwei Schritten soll er bis Januar } 2020 \text { weiter } \\
\text { steigen und dann 11,35 Euro pro Stunde im Westen und } \\
\text { 10,85 Euro im Osten betragen. Bereits im April } 2017 \\
\text { verständigte sich die Pflegekommission aus Arbeit- } \\
\text { gebern und Arbeitnehmern auf die Erhöhung. Der Pfle- } \\
\text { gemindestlohn gilt für alle Betriebe, die ambulante, } \\
\text { teilstationäre oder vollstationäre Pflegeleistungen oder } \\
\text { ambulante Krankenpflegeleistungen erbringen. }\end{array}$ \\
\hline
\end{tabular}


Krankenhauspolitische Chronik

\begin{tabular}{|c|c|c|c|}
\hline Termin & Leitbegriff & Vorgang & Legende \\
\hline 17. Juli 2017 & Politik & $\begin{array}{l}\text { CSU-Programm zur } \\
\text { Bundestagswahl } \\
\text { "Bayernplan" }\end{array}$ & $\begin{array}{l}\text { Die CSU hat ihr Wahlprogramm veröffentlicht. Kranken- } \\
\text { hauspolitisch relevant sind folgende Vorhaben: Kranken- } \\
\text { häuser wohnortnah sichern, Investitionen bundesweit } \\
\text { erhöhen, Preisentwicklung bei der Krankenhausvergü- } \\
\text { tung berücksichtigen - vor allem durch vollen Ausgleich } \\
\text { der Tarifsteigerung, Krankenhausstrukturfonds zur Ver- } \\
\text { besserung der IT-Sicherheit und Stärkung der sektoren- } \\
\text { übergreifenden Versorgung fortsetzen, Verbesserungen } \\
\text { für Hebammen auch auf Belegärzte übertragen, verbes- } \\
\text { serten Haftungsschutz für Hauptabteilungen Gynäkolo- } \\
\text { gie und Geburtshilfe, Rahmenbedingungen für Pflege- } \\
\text { personal weiter verbessern und Attraktivität steigern. }\end{array}$ \\
\hline 11. Juli 2017 & $\begin{array}{l}\text { Wissen- } \\
\text { schaft }\end{array}$ & $\begin{array}{l}\text { Roland Berger ver- } \\
\text { öffentlicht „Kranken- } \\
\text { hausstudie 2017“ }\end{array}$ & $\begin{array}{l}\text { Obwohl knapp } 90 \text { Prozent der deutschen Kliniken eine } \\
\text { Digitalstrategie entwickelt haben, ist die Umsetzung und } \\
\text { der Aufbau der notwendigen IT-Kapazitäten mangelhaft, } \\
\text { weil die nötigen finanziellen Mittel fehlen. Laut Studie } \\
\text { hat sich die wirtschaftliche Lage im vergangenen Jahr } \\
\text { erneut zugespitzt. Trotz steigender Umsätze konnten } \\
\text { über } 40 \text { Prozent der Krankenhäuser keinen Überschuss } \\
\text { erwirtschaften. }\end{array}$ \\
\hline 10. Juli 2017 & Politik & $\begin{array}{l}\text { BMBF plant bundes- } \\
\text { weite Medizindaten- } \\
\text { bank }\end{array}$ & $\begin{array}{l}\text { Das Bundesministerium für Bildung und Forschung } \\
\text { (BMBF) will eine deutschlandweite Medizindatenbank } \\
\text { aufbauen. Ziel ist es, eine bessere Gesundheitsforschung } \\
\text { und Patientenversorgung zu ermöglichen. Dafür sollen } \\
\text { die wachsenden Datenschätze - von Röntgenbildern bis } \\
\text { hin zu Erbgut-Analysen - in einer nationalen Infrastruk- } \\
\text { tur verknüpft werden. }\end{array}$ \\
\hline 7. Juli 2017 & $\begin{array}{l}\text { Selbstver- } \\
\text { waltung }\end{array}$ & $\begin{array}{l}\text { Pflegestellen-Förder- } \\
\text { programm schuf } 2016 \\
\text { mehr als } 1.600 \text { zusätz- } \\
\text { liche Pflegestellen }\end{array}$ & $\begin{array}{l}\text { Der GKV-SV hat am } 30.06 .2017 \text { den Bericht über die } \\
\text { Umsetzung des Pflegestellen-Förderprogramms im Jahr } \\
2016 \text { an das Bundesministerium für Gesundheit (BMG) } \\
\text { übermittelt. Der Bericht belegt, dass die GKV den Kran- } \\
\text { kenhäusern in diesem Zeitraum zusätzliche Finanzmittel } \\
\text { in Höhe von ca. 52,3 Mio. Euro zur Verfügung gestellt } \\
\text { hat. Von Jahresabschlussprüfern bestätigte Angaben } \\
\text { liegen noch nicht vor. Fast } 600 \text { Krankenhäuser erhielten } \\
\text { durch das aktuelle Pflegestellen-Förderprogramm } \\
\text { zusätzliche Mittel, um mehr Personal einzustellen. Ins- } \\
\text { gesamt wurden } 2016 \text { mit den Krankenkassen etwa } \\
1.410 \text { neue Stellen vereinbart. Hinzu kommen etwa } \\
227 \text { Pflegepersonalstellen, die sich aus Hochrechnungen } \\
\text { ergaben. }\end{array}$ \\
\hline 7. Juli 2017 & $\begin{array}{l}\text { Selbstver- } \\
\text { waltung }\end{array}$ & $\begin{array}{l}\text { Vereinbarung für } \\
\text { klinische Sektionen: } \\
750 \text { Euro pro } \\
\text { Obduktion }\end{array}$ & $\begin{array}{l}\text { Die Vertragsparteien auf Bundesebene haben sich auf } \\
\text { eine Obduktionsvereinbarung verständigt, die rückwir- } \\
\text { kend ab dem } 01.01 .2017 \text { die Zuschlagsfinanzierung von } \\
\text { Obduktionen in Krankenhäusern regelt. Klinische Sek- } \\
\text { tionen werden mit einem Zuschlag von jeweils } 750 \text { Euro } \\
\text { finanziert, sofern das Krankenhaus eine indikationsbe- } \\
\text { zogene Obduktionsrate erfüllt. Die Kliniken müssen } \\
\text { bestimmte Qualitätsstandards erfüllen. Für die Gewäh- } \\
\text { rung des Zuschlags gilt eine bestimmte indikationsspezi- } \\
\text { fische Obduktionsrate. Diese liegt } 2017 \text { bei 7,5 Prozent, } \\
2018 \text { bei } 10 \text { Prozent und ab } 2019 \text { bei 12,5 Prozent. }\end{array}$ \\
\hline
\end{tabular}




\begin{tabular}{|c|c|c|c|}
\hline \multicolumn{4}{|c|}{ Krankenhauspolitische Chronik } \\
\hline Termin & Leitbegriff & Vorgang & Legende \\
\hline 7. Juli 2017 & Politik & $\begin{array}{l}\text { Bundesrat stimmt } \\
\text { Pflegeberufereform- } \\
\text { gesetz zu }\end{array}$ & $\begin{array}{l}\text { Der Bundesrat hat dem Gesetz zur Reform der Pflegeaus- } \\
\text { bildung zugestimmt, das der Bundestag am 22. Juni } \\
\text { verabschiedet hatte. Von } 2020 \text { an wird die Ausbildung } \\
\text { in den drei Berufsfeldern Krankenpflege, Kinderkranken- } \\
\text { pflege und Altenpflege verzahnt. Dadurch erhalten } \\
\text { die Auszubildenden die Möglichkeit, zwischen den } \\
\text { Bereichen zu wechseln. }\end{array}$ \\
\hline 5. Juli 2017 & Politik & $\begin{array}{l}\text { Fischbach ist neue } \\
\text { Patienten- und Pflege- } \\
\text { beauftragte }\end{array}$ & $\begin{array}{l}\text { Ingrid Fischbach ist die neue Beauftragte der Bundesre- } \\
\text { gierung für die Belange der Patientinnen und Patienten } \\
\text { sowie Bevollmächtigte für Pflege. Die CDU-Politikerin } \\
\text { übernimmt das Amt von Karl-Josef Laumann. Laumann } \\
\text { ist seit Anfang Juli neuer Sozial- und Gesundheitsminister } \\
\text { in Nordrhein-Westfalen (NRW). }\end{array}$ \\
\hline 3. Juli 2017 & Politik & $\begin{array}{l}\text { Union: Regierungs- } \\
\text { programm } 2017 \text { bis } \\
2021 \text { beschlossen }\end{array}$ & $\begin{array}{l}\text { CDU und CSU haben ihr Regierungsprogramm „Für ein } \\
\text { Deutschland, in dem wir gut und gerne leben“ beschlos- } \\
\text { sen. Krankenhauspolitisch relevant sind insbesondere } \\
\text { folgende Punkte: Berücksichtigung der Preisentwicklung } \\
\text { bei der Krankenhausvergütung mit einem vollen } \\
\text { Ausgleich der Tarifsteigerung, Verbesserung der Arbeits- } \\
\text { bedingungen der Pflegekräfte, Sicherung einer gut } \\
\text { erreichbaren Krankenhausversorgung in der Fläche und } \\
\text { Stärkung der Zusammenarbeit mit spezialisierten Kli- } \\
\text { niken und Universitätskliniken. }\end{array}$ \\
\hline 1. Juli 2017 & $\begin{array}{l}\text { Selbstver- } \\
\text { waltung }\end{array}$ & $\begin{array}{l}\text { Neue Psych-Personal- } \\
\text { nachweis-Vereinba- } \\
\text { rung in Kraft }\end{array}$ & $\begin{array}{l}\text { GKV-SV, PKV und DKG haben sich auf die Psych-Personal- } \\
\text { nachweis-Vereinbarung verständigt. Sie regelt die Nach- } \\
\text { weispflichten zur Umsetzung der Psych-PV für die Jahre } \\
2016 \text { bis } 2019 \text { und beinhaltet insbesondere Vorgaben } \\
\text { zum Geltungsbereich der Nachweispflicht, zum Umfang } \\
\text { des für den Nachweis relevanten Personals sowie zum } \\
\text { Übermittlungsverfahren und zu den Übermittlungs- } \\
\text { fristen. }\end{array}$ \\
\hline
\end{tabular}

Krankenhaus-Report 2019

Die Krankenhauspolitische Chronik ab dem Jahr 2000 bis Juni 2017 finden Sie unter

> https://doi.org/10.1007/978-3-662-58225-1.

Open Access Dieses Kapitel wird unter der Creative Commons Namensnennung 4.0 International Lizenz(http://creativecommons. org/licenses/by/4.0/deed.de) veröffentlicht, welche die Nutzung, Vervielfältigung, Bearbeitung, Verbreitung und Wiedergabe in jeglichem Medium und Format erlaubt, sofern Sie den/die ursprünglichen Autor(en) und die Quelle ordnungsgemäß nennen, einen Link zur Creative Commons Lizenz beifügen und angeben, ob Änderungen vorgenommen wurden.

Die in diesem Kapitel enthaltenen Bilder und sonstiges Drittmaterial unterliegen ebenfalls der genannten Creative Commons Lizenz, sofern sich aus der Abbildungslegende nichts anderes ergibt. Sofern das betreffende Material nicht unter der genannten Creative Commons Lizenz steht und die betreffende Handlung nicht nach gesetzlichen Vorschriften erlaubt ist, ist für die oben aufgeführten Weiterverwendungen des Materials die Einwilligung des jeweiligen Rechteinhabers einzuholen.

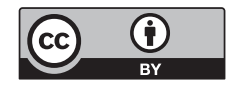

